\title{
An Extension to Artifact-free Projection Overlaps
}

\author{
Jianyu Lin \\ Department of Electrical and Computer Engineering \\ Curtin University, GPO Box U1987 \\ Perth, WA 6845, Australia
}

Purpose: In multi-pinhole SPECT, the overlapping of projections has been used to increase sensitivity. Avoiding artifacts in the reconstructed image associated with projection overlaps (multiplexing) is a critical issue. In our previous report, two types of artifact-free projection overlaps, i.e. projection overlaps that do not lead to artifacts in the reconstructed image, were formally defined and proved, and were validated via simulations. In this work, a new proposition is introduced to extend the previously defined type-II artifact-free projection overlaps so that a broader range of artifact-free overlaps is accommodated. One practical purpose of the new extension is to design a baffle-window multi-pinhole system with artifact-free projection overlaps.

Methods: First, the extended type-II artifact-free overlap was theoretically defined and proved. The new proposition accommodates the situation where the extended type-II artifact-free projection overlaps can be produced with incorrectly reconstructed portions in the reconstructed image. Next, to validate the theory, the extended-type-II artifact-free overlaps were employed in designing the multiplexing multi-pinhole spiral orbit imaging systems with a baffle window. Numerical validations were performed via simulations, where the corresponding 1-pinhole non-multiplexing reconstruction results were used as the benchmark for artifact-free reconstructions. The mean square error (MSE) was the metric used for comparisons of noise-free reconstructed images. Noisy reconstructions were also performed as part of the validations.

Results: Simulation results show that for noise-free reconstructions, the MSEs of the reconstructed images of the artifact-free multiplexing systems are very similar to those of the corresponding 1pinhole systems. No artifacts were observed in the reconstructed images. Therefore, the testing results for artifact-free multiplexing systems designed using the extended type-II artifact-free overlaps numerically validated the developed theory.

Conclusions: First, the extension itself is of theoretical importance because it broadens the selection range for optimizing multiplexing multi-pinhole designs. Second, the extension has an immediate application: using a baffle window to design a special spiral orbit multi-pinhole imaging system with projection overlaps in the orbit axial direction. Such an artifact-free baffle-window design makes it possible for us to image any axial portion of interest of a long object with projection overlaps to increase sensitivity.

Key words: multi-pinhole SPECT, artifact in the reconstructed image, projection overlap, multiplexing 
Pinhole collimation is widely used in single photon emission computed tomography (SPECT). To increase the sensitivity, a major issue in SPECT, multiple pinhole collimations with projection overlaps are employed. Overlaps in the projections make use of the detector area more than once, or equivalently, in the overlapped region the sensor receives photons from more than one pinhole. As a result, the overall sensitivity is increased.

Although there are many studies on multi-pinhole collimation and on the increased sensitivity associated with projection overlaps, ${ }^{1,11-13}$ a controversial issue has been whether the increased sensitivity contributes to the final quality of the reconstructed image, such as signal to noise ratio, level of artifacts, etc.

The prevailing view from previous studies has been that the overall image quality may not improve by introducing overlaps in the projections. Many researchers are sceptical about the use of projection overlaps. Indeed, although there are cases where a high degree of multiplexing (e.g. Meikle et al. ${ }^{19}$ ) was used, there are also cases where the system was designed with no overlap at all (Beekman et al. ${ }^{20}$ ). Many people would believe that once the detector area was fully utilized, the extra sensitivity from projection overlaps could only compensate for the ambiguity posed by the overlaps, ${ }^{1}$ and that projection overlaps would degrade the reconstructed image quality because of the unavoidable artifacts associated with the overlaps, especially for non-sparse phantoms. ${ }^{11}$

Recently, in a study ${ }^{2,18}$ on slit-slat collimators, it was experimentally shown that artifacts in the reconstructed image associated with projection overlaps could be removed with the help of an extra complete data set of non-overlapped projections. In Reference 3, for the first time, projection overlaps that do not lead to artifacts in the reconstructed images were formally defined and proved for pinhole collimation. Consequently, for multiplexed multi-pinhole SPECT systems, projection overlaps that do not lead to reconstruction artifacts were termed 'artifact-free projection overlaps' to distinguish them from other projection overlaps.

In both of the above studies, it was shown there were designed system configurations where the artifact-free overlaps could significantly contribute to the final quality (contrast to noise ratio) of the reconstructed image. However, unlike the tidy definitions of artifact-free projection overlaps, there are still no theoretical rules for identifying the configurations with which artifact-free overlaps contribute to the noise reduction of the reconstructed image. ${ }^{3}$ The contribution from the overlapped region to noise reduction in the reconstructed image depends upon many factors, such as the adjacent pinhole distance, the amount of the non-overlapped area, and the configuration of the overlaps, etc. This means that in order to find a good design or an empirical design rule for making use of the overlaps, various artifact-free overlapping configurations must be tested via extensive simulations.

Therefore, there are two research directions for further explorations of making use of the artifactfree projection overlaps. One is to increase the range of the artifact-free overlaps such that more configurations are provided for the simulation-based selection process. In the previous report ${ }^{3}$, only two types of artifact-free overlaps were introduced. With increased types of artifact-free overlaps, there is a greater chance of obtaining good designs from the combinations of the larger varieties of configurations. The other research direction is the simulation-based selections among the possible good configurations. This study focuses on the former: to provide a broader range of artifact-free 
projection overlaps. Specifically, we propose an extension to the artifact-free projection overlaps presented in Reference 3.

The proposed extension in this paper also has immediate application of practical interest. Recall a strong conclusion in the previous report ${ }^{3}$ : when a spiral orbit, with a small enough orbit pitch and a large enough axial orbit range, is used, artifact-free reconstruction is possible for any projection overlaps produced by pinholes aligned along the axial direction of the spiral orbit. This makes it possible to employ the axially-aligned overlaps. However, the "large enough axial orbit range" suggests that the axial range of the spiral orbit needs to be much longer than the axial dimension of the imaged object so that the entire object is imaged and reconstructed. ${ }^{3}$ This becomes impractical. In reality, we often encounter situations where the object is long, but only a small portion must be imaged. For example, when imaging the human brain, we would never want to scan the entire body. An immediate solution for imaging a small portion of a long object seems to be the addition of blocking baffles. The baffles would block the portions that do not need to be imaged, and thus the small portion that needs to be imaged will be projected through a baffle window. However, when baffles are applied, the required condition for the axially-aligned artifact-free overlaps specified in Reference 3 is not met (details are shown later). The proposed extension in this paper accommodates the artifact-free projection overlaps produced with a baffle window, which is of particular importance when employing axially-aligned artifact-free overlaps in imaging and reconstructing a small portion of a long object.

\section{THEORY}

\section{A. Background and Terminology}

First, we present the background and terminologies used in developing the theory of artifact-free projection overlaps, which are important but were not clarified in Reference 3.

In pinhole tomographic imaging, an object $V_{0}$ is projected through several pinholes, and a collection of projection images is obtained:

$F\left(V_{0}\right)=\left\{\mathrm{PI}_{p}, p \in \mathrm{P}\right\}$,

where function $F(\cdot)$ represents the projection operation, $\mathrm{PI}_{p}$ is the projection image along direction $p$, and set $\mathrm{P}$ is a collection of projection directions, which can be continuous and infinite in theory. A multiple pinhole projection image is the sum of the individual projection images from each pinhole. More precisely, at a projection direction $p$,

$\mathrm{PI}_{p}=\sum_{n=1}^{N} \mathrm{IPI}_{p, n}$

where $N$ is the total number of pinholes, $\operatorname{IPI}_{p, n}$ is the individual projection image in the projection image $\mathrm{PI}_{p}$ through pinhole $n$. The task of image reconstruction is to find $V$, the solution of (1), given the projection set $\left\{\mathrm{PI}_{p}\right\}$.

Theoretically, for the single pinhole condition, when the projection set $\left\{\mathrm{PI}_{p}\right\}$ satisfies the sufficient conditions, ${ }^{14,15}$ an exact reconstruction, i.e. the reconstructed image $V=V_{0}$, is guaranteed and the 
analytical inversion formulas for $V$ exist. ${ }^{14,15}$

In reality, such "exactness" is very difficult (if not impossible) to numerically verify even to computer precision. This is because integrations in the inversion formulas need unreasonably high computational power to achieve the precision. Besides the computational difficulty, real acquired data can never be ideal. The pinholes used are of finite sizes, which are normally not relatively small. The projections $\mathrm{PI}_{p}$ are sampled (not continuous), and the pixel size is also normally not relatively small. Therefore, the term "exact reconstruction" here is reserved for only the theoretical exact inversion formulas of $V_{0}$, such as the ones shown in the existing literatures. ${ }^{14,15}$

Practical reconstruction algorithms include analytical reconstruction algorithms and iterative reconstruction algorithms. Compared with the analytical reconstruction algorithms, statisticallybased iterative reconstruction algorithms provide improved image quality with more accurate physical and statistical modelling of gamma photon detection processes in SPECT cameras. Among the statistical iterative reconstruction algorithms, the Maximum Likelihood Expectation Maximization (MLEM) algorithm is well-developed and is widely used. ${ }^{16,17}$

In iterative reconstruction algorithms, linear projections (1) are decretized into the format

$$
g=H f
$$

where $f$ is a long vector whose elements are the image voxels, $g$ is also a long vector whose elements are the pixels from all the projections, and $H$ is a huge matrix that describes the system. When the randomness in projections $g$ is considered, (3) should be

$$
E[g]=H f,
$$

where $E[\cdot]$ denotes expected value. The MLEM algorithm provides the statistical estimate of $f$ from data $g$.

Suppose for a system, the projection set $\left\{\mathrm{PI}_{p}\right\}$ is sufficient ${ }^{14,15}$ to reconstruct $V_{0}$. Then, the solution of (1) is unique and equal to $V_{0}$. Since (3) is a discrete version of (1), $g, f$ and $H$ in (3) correspond to $\left\{\mathrm{PI}_{p}\right\}, V_{0}$ and $F(\cdot)$ in (1). Therefore, solution $f$ of (3) is a sampled version of $V_{0}$ and is artifact-free. Since the EM estimator (MLEM) is unbiased, the mean of the estimated noisy images from the noisy data is equal to the image obtained from the noiseless data. Therefore, to numerically validate the reconstruction correctness of (1) when $V_{0}$ can be exactly reconstructed, we need to simulate only the noiseless version of $f$ and $g$. (Note, as already mentioned, that such a numerical validation is not an exact reconstruction.)

Suppose the projection set $\left\{\mathrm{PI}_{p}\right\}$ is not sufficient to reconstruct $V_{0}$. There may exist many solutions to (1). The non-uniqueness makes the solution to (1) ambiguous. In other words, the inversion of $V_{0}$ from $\left\{\mathrm{PI}_{p}\right\}$ is impossible. Correspondingly, equation (3), the discrete version of (1), also has the same ambiguity, and the reconstructed image is inaccurate even with noiseless data. In this case, we see artifacts or errors in the reconstructed image $f$. Note, we are interested in the reconstruction errors from the system $F(\cdot)$ in (1) or $H$ in (3), noise in the reconstructed image associated with the statistical noise in data is not categorized as artifacts. Therefore, to observe the artifacts associated with a system, noiseless data provides the clearest view, because the noiseless reconstructed image is the mean of the noisy reconstructed images, and when noise exists, small artifacts will be 
masked. For this reason, in many existing literatures noiseless simulations have been employed to check artifacts. ${ }^{1,18}$

Therefore, in finding artifact-free projection overlaps, we first theoretically prove that exact reconstruction holds for (1). Then, we validate it numerically via simulations using the discrete version system description - equation (3), where the corresponding 1-pinhole reconstruction results are the benchmark of artifact-free reconstruction, and mean square error is the metric for comparisons. Simulations are performed using MLEM with noiseless and noisy data. The noiseless reconstructions provide the mean of noisy situations, and are sufficient to observe any artifacts from the system matrix $H$ in (3). The noisy simulations are used to validate the assertion.

\section{B. Summary of the Two Types of Artifact-free Projection Overlaps}

To introduce the extension and to familiarize readers with artifact-free projection overlaps, in this subsection, we briefly summarize the two types of artifact-free overlaps defined in Reference 3.

Definition 1: For a particular pinhole, say pinhole $i(n=i)$ in a multi-pinhole imaging system, if the collection of the non-overlapped regions in $\operatorname{IPI}_{p, i}(p \in \mathrm{P})$ is sufficient to reconstruct a fraction of the imaged object $V_{0}$, then, the overlaps in $\operatorname{IPI}_{p, i}(p \in \mathrm{P})$ are defined as type-I artifact-free overlaps.

Proposition 1: If type-I artifact-free overlaps exist for a fraction of $V_{0}$ in $\operatorname{IPI}_{p, i}(p \in \mathrm{P})$ for any pinhole $i$, then, that fraction can be reconstructed free from artifacts regardless of the existence of other overlaps in other $\operatorname{IPI}_{p, n}(n \neq i, p \in \mathrm{P})$.

Note, type-I artifact-free overlaps are defined to appear collectively in a set of projections in which the non-overlapped regions are sufficient to reconstruct the object fraction.

Proposition 1 was proved, ${ }^{3}$ and examples of type-I artifact-free overlaps were provided. ${ }^{3}$ The intuitive explanation is that if the non-overlapped regions alone can reconstruct the object correctly (such as the examples used in Reference 4), then, all the overlaps are simply consistent with the sufficient non-overlapped regions (for details, see Reference 3).

Definition 2: Assume a portion of an overlap consists of IPIs from $K$ pinholes. Within that overlap portion, if out of the $K$ IPIs there are $(K-1)$ IPIs, each of which is projected from a fraction of the object that can be exactly reconstructed, then, this overlap portion is defined as a type-II artifactfree overlap.

Proposition 2: A type-II artifact-free overlap is equivalent to nonoverlapped regions for its contributing IPIs in reconstruction.

Proposition 2 was also proved. ${ }^{3}$ Fig. 1 shows an example of a type-II artifact-free overlap from axially-aligned pinholes. Let $V$ be the reconstructed image - the solution of (1), and $V_{0}$ be the original object as usual. Denote the projections from $V \mathrm{PI}_{p}^{\prime}$ and $\mathrm{IPI}_{p, n}^{\prime}$, and the projections from $V_{0}$ $\mathrm{PI}_{p}$ and IPI ${ }_{p, n}$. In Fig. 1(a), the detector is at the starting point of a scanning spiral orbit ( $p=0$, i.e. the 0th projection), and the projection through pinhole 1 has a non-overlapped region AC. As 
the spiral scan progresses to the left, the non-overlapped regions of AC vary. As long as the pitch of the scanning spiral orbit is small enough, a collection of these non-overlapped regions AC ( $p=0,1, \ldots, r$, for some $r$ ) is sufficient to reconstruct a portion of the object, which is colored green in Fig. 1(a). Now, from Fig. 1(b) it can be seen that, at the starting point of the scan orbit $(p=0)$, the projection through pinhole 2 contributing to overlap $\mathrm{CB}$ is from an object fraction $V_{r}$ that can be perfectly reconstructed. Notice that overlap CB is formed by only two IPIs: $\mathrm{PI}_{0}(\mathrm{CB})=\mathrm{IPI}_{0,1}(\mathrm{CB})+$ $\mathrm{IPI}_{0,2}(\mathrm{CB})$. For the reconstructed volume $V, \mathrm{PI}_{0}^{\prime}(\mathrm{CB})=\mathrm{PI}_{0}(\mathrm{CB})$, because $V$ is a solution of Equation (1); its individual projection image $\operatorname{IPI}_{0,2}^{\prime}(\mathrm{CB})=\operatorname{IPI}_{0,2}(\mathrm{CB})$, because $\operatorname{IPI}_{0,2}^{\prime}(\mathrm{CB})$ is projected from a fraction in $V$ that is identical to $V_{0}$. Then, $\operatorname{IPI}_{0,1}^{\prime}(\mathrm{CB})$ from $V$ is forced to be equal to $\operatorname{IPI}_{0,1}(\mathrm{CB})$ from $V_{0}$, because $\mathrm{IPI}_{0,1}^{\prime}(\mathrm{CB})=\mathrm{PI}_{0}^{\prime}(\mathrm{CB})-\mathrm{IPI}_{0,2}^{\prime}(\mathrm{CB})=\mathrm{PI}_{0}(\mathrm{CB})-\mathrm{IPI}_{0,2}(\mathrm{CB})$ $=\mathrm{IPI}_{0,1}(\mathrm{CB})$. Therefore, for any reconstructed image $V$, overlap $\mathrm{CB}$ is equivalent to nonoverlapped regions as if its two contributing IPIs from pinhole 1 and 2 were recorded on two separate sensors: $\operatorname{IPI}_{0,1}^{\prime}(\mathrm{CB})=\operatorname{IPI}_{0,1}(\mathrm{CB})$ and $\mathrm{IPI}_{0,2}^{\prime}(\mathrm{CB})=\mathrm{IPI}_{0,2}(\mathrm{CB})$.

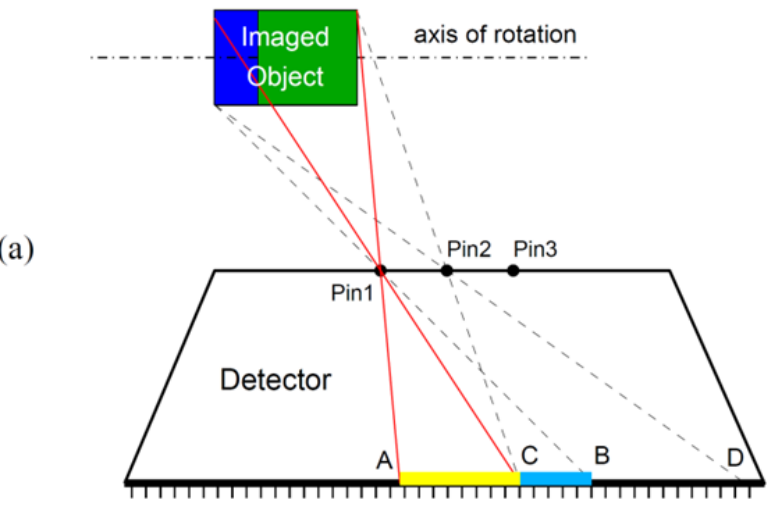

(b)
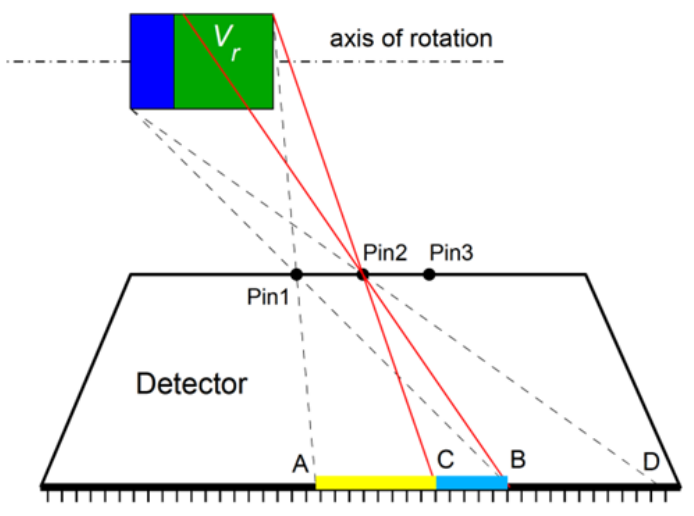

Fig. 1. Sketches for a possible formation of a type-II artifact-free overlap: (a) The collection of the non-overlapped regions AC is sufficient to reconstruct a portion of the object (shaded green). (b) Region CB of the IPI through pinhole 2 is from the object fraction $V_{r}$ that can be perfectly reconstructed.

As a consequence, the following very strong conclusion is drawn from the type-II artifact-free overlaps: A finite size object imaged by a spiral orbit system with projection overlaps from a finite number of axially aligned pinholes can always be reconstructed free from artifacts, if the pitch of 
the spiral orbit is small enough and the axial range of the spiral orbit is large enough. This is understandable. Suppose the spiral orbit satisfies the requirements. Then, the collection of the nonoverlapped regions in the projections near the starting point of the scanning orbit $(p=0,1, \ldots, r$ for some $r$ ) or the collection of the non-overlapped regions in the projections near the ending point of the scanning orbit $(p=s, s+1, \ldots, P-1$ for some $s$ ) is sufficient to reconstruct part of the object as illustrated earlier in Fig. 1. Type-II artifact-free overlaps will form sequentially (see Iterative Artifact Checking Principle). ${ }^{3}$ Eventually, the equivalent non-overlapped regions (progressively released by the type-II artifact-free overlaps) are sufficient to reconstruct the entire object, and no artifact is expected for the entire reconstructed object.

\section{C. The Extended Type-II Artifact-free Overlap}

As mentioned in the Introduction, using type-II artifact-free overlaps to image and reconstruct the entire object is not ideal for the situation where only a small portion of a long object needs to be imaged, and a possible solution could be the application of a baffle window.

(a)

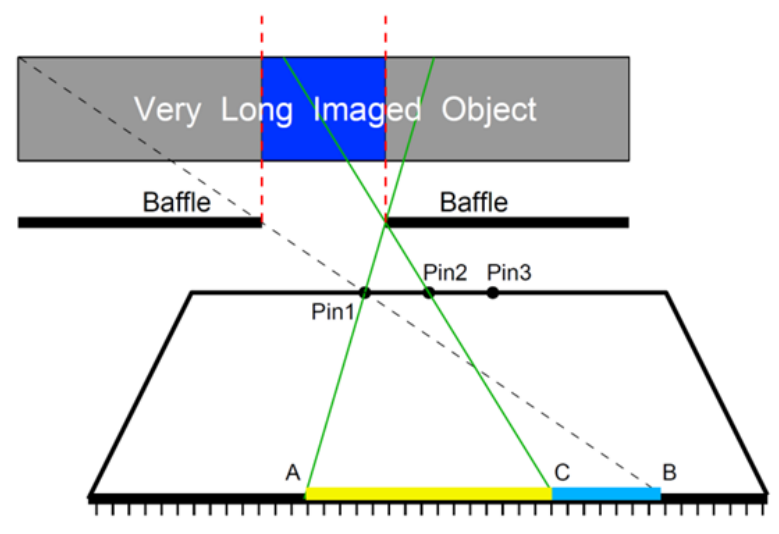

(b)

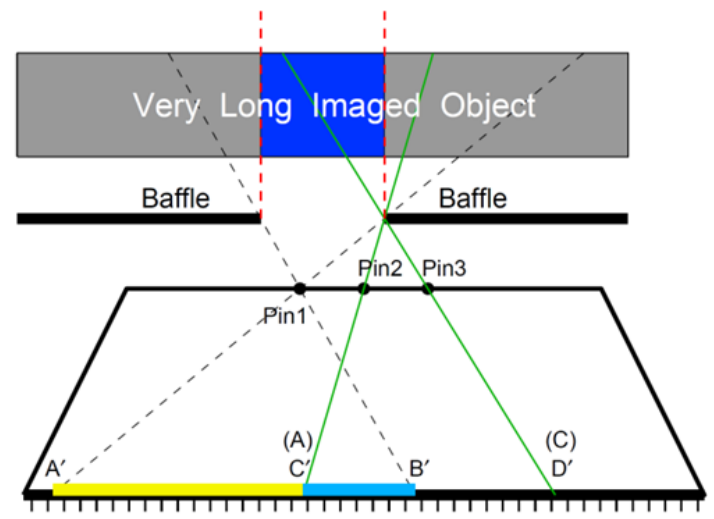

Fig. 2. Formation of an extended type-II artifact-free overlap with a baffle window: (a) The entire projection from the reconstructed image $V$ is identical to that from the original object $V_{0}$ even if $V \neq V_{0}$. Note the reconstructed volume, which determines the size of $V$, should be large enough to include all the regions in $V_{0}$ contributing to the scanned projections. (b) At the corresponding position of (a) in the next turn of the spiral orbit, the contribution to overlap $\mathrm{C}^{\prime} \mathrm{B}^{\prime}$ from pinhole 2 is equal to the corresponding projection through pinhole 1 in (a), and thus equal to the projection from the original object $V_{0}$. By definition, overlap $\mathrm{C}^{\prime} \mathrm{B}^{\prime}$ through pinhole 1 is an extended type-II artifact-free overlap. 
In Fig. 2, baffles are applied to produce a window. The axial position of the window does not change during the entire spiral orbit scan. (In real systems, a spiral orbit is normally generated by moving the bed. There, the window must move along with the bed.) Suppose a spiral orbit is used; then, with a single pinhole there can be an artifact-free reconstructed portion within the blue shaded region of the object. (In the extreme case, when the pitch of the spiral orbit is infinitely small, the entire blue region can be reconstructed with no artifact.) However, the regions outside, shaded grey in Fig. 2, cannot be perfectly reconstructed, but they do contribute to overlaps. As a result, the type-II artifact-free overlaps do not apply to the baffle windowing situation here. Therefore, the type-II artifact-free overlap is extended to accommodate a wider range of situations.

Definition 2ext: Suppose a portion of an overlap consists of projections from $K$ pinholes. Let $V$ be a reconstructed image - any solution of Equation (1), and $V_{0}$ be the original object ( $V \neq V_{0}$ regions may exist). Within the overlap portion, if the projection of $V$ through $(K-1)$ of the $K$ pinholes is equal to the projection of $V_{0}$ through the same $(K-1)$ pinholes, then, on the IPI of the remaining pinhole, this overlap portion is defined as an extended type-II artifact-free overlap.

Proposition 2ext: An extended type-II artifact-free overlap is equivalent to a non-overlapped region in reconstruction.

Proof: $V$ is a solution of Equation (1). Denote its projections as $\mathrm{PI}_{p}^{\prime}$ and its individual projection images as $\mathrm{IPI}_{p, n}^{\prime}$. The projections of $V_{0}$ are denoted as $\mathrm{PI}_{p}$ and $\mathrm{IPI}_{p, n}$ as usual. By definition, the overlap consists of $K$ IPIs; e.g., at projection $p=q$, the overlap in $\mathrm{PI}_{q}$ consists of $\operatorname{IPI}_{q, k}, k=1,2$, $\ldots, K$. And for the projection through $(K-1)$ of the $K$ pinholes, say $k=1,2, \ldots,(K-1)$ without loss of generality, $\sum_{k=1}^{K-1} \mathrm{IPI}_{q, k}^{\prime}=\sum_{k=1}^{K-1} \mathrm{IPI}_{q, k}$ holds within the overlap. Then, for the IPI through pinhole $k=K$, we have

$\mathrm{IPI}_{q, K}^{\prime}=\mathrm{IP}_{q}^{\prime}-\sum_{k=1}^{K-1} \mathrm{IPI}_{q, k}^{\prime}=\mathrm{IP}_{q}-\sum_{k=1}^{K-1} \mathrm{IPI}_{q, k}=\mathrm{IPI}_{q, K}$

within the overlap, where we have used $\mathrm{IP}_{q}^{\prime}=\mathrm{IP}_{q}$ because $V$ is a solution of Equation (1). Therefore, for pinhole $K$, within the extended type-II artifact-free overlap, $\mathrm{IPI}_{q, K}^{\prime}=\mathrm{IPI}_{q, K}$. That is, the overlap is equivalent to a non-overlapped region for pinhole $K$ as if the IPI projected from pinhole $K$ is recorded on a separate sensor.

\section{D. An Application of the Extended Type-II Artifact-free Overlap}

The proof of the proposition is straightforward, and is similar to the previous proof of the type-II artifact-free overlaps. ${ }^{3}$ However, the extended type-II artifact-free overlap includes a much wider range of artifact-free configurations than the original type-II artifact-free overlap. In fact, the demanding part is not the proving of the proposition. Rather, the reconstruction of a system that uses the proposition involves some difficult logics and 3-dimensional geometry that need abstract imagination. The baffle-window design for imaging a small portion of a long object is a good example, which will be discussed next. We also want to point out that the extended type-II artifactfree overlaps can be configured in numerous circumstances for both axially aligned pinhole 
multiplexing and trans-axially aligned pinhole multiplexing. However, in this paper, we focus on the axially-aligned pinholes in a baffle window imaging system, as this is a practical situation of imaging a small axial portion of a long object.

In Fig. 2(a), the detector is at an orbit point near the starting point of a spiral scan, assuming the spiral scan is from right to left in the figure. To produce the extended type-II artifact-free overlaps, we set the pitch of the spiral orbit to be equal to the distance of the adjacent pinholes. In Fig. 2(b), the detector is at the corresponding position of Fig. 2(a) in the next turn of the spiral orbit. Thus, in Fig. 2(b), pinhole 2 is at the pinhole 1 position in Fig. 2(a) and pinhole 3 is at the pinhole 2 position in Fig. 2(a). Therefore, the IPI through pinhole 2 in Fig. 2(b) should be equal to the IPI through pinhole 1 in Fig. 2(a). Thus, for the reconstructed image $V$,

$\mathrm{IPI}_{\text {pin1@Fig2a }}^{\prime}=\mathrm{IPI}_{\text {pin2@Fig2b }}^{\prime}$,

and for the original object $V_{0}$,

$\mathrm{IPI}_{\text {pin1@Fig2a }}=\mathrm{IPI}_{\text {pin2@Fig2b }}$.

On the other hand, in Fig. 2(a) the entire projection $\mathrm{PI}_{@ \text { Fig2a }}^{\prime}$ from the reconstructed image $V$ is equal to the entire projection $\mathrm{PI}_{\varrho \text { Fig2a }}$ from the original object $V_{0}$, because $V$ is one of the non-unique solutions of Equation (1). Thus, as part of the entire projection, for region AC through pinhole 1 in Fig. 2(a), we have

$\mathrm{IPI}_{\text {pin1@Fig2a }}^{\prime}(\mathrm{AC})=\mathrm{IPI}_{\text {pin1@Fig2a }}(\mathrm{AC})$.

It can be seen region AC in Fig. 2(a) corresponds to region C'D' in Fig. 2(b). Substitute (6) into the left-hand side of (8), and (7) into the right-hand side of (8), we have

$\operatorname{IPI}_{\text {pin2@Fig2b }}^{\prime}\left(\mathrm{C}^{\prime} \mathrm{D}^{\prime}\right)=\mathrm{IPI}_{\text {pin2@Fig2b }}\left(\mathrm{C}^{\prime} \mathrm{D}^{\prime}\right)$.

Following (9), for the overlap $\mathrm{C}^{\prime} \mathrm{B}$ ', which is a portion of $\mathrm{C}^{\prime} \mathrm{D}$ ', the projection of $V$ through pinhole 2 is equal to the projection of $V_{0}$ through pinhole 2 , or

$$
\mathrm{IPI}_{\text {pin2@Fig2b }}^{\prime}\left(\mathrm{C}^{\prime} \mathrm{B}^{\prime}\right)=\mathrm{IPI}_{\text {pin2@Fig2b }}\left(\mathrm{C}^{\prime} \mathrm{B}^{\prime}\right) \text {. }
$$

Now, overlap C'B' in Fig. 2(b) comprises IPIs from two $(K=2)$ pinholes: pinhole 1 and pinhole 2. Equation (10) states that for one $(K-1=1)$ of the two pinholes, pinhole 2, its IPI from the reconstructed image $V$ is equal to that from the original object $V_{0}$. Then, by definition, in the IPI of the remaining pinhole, pinhole 1, in Fig. 2(b), overlap $C^{\prime} B^{\prime}$ is an extended type-II artifact-free overlap, which is equivalent to a non-overlapped region. Thus, the whole IPI of pinhole 1 in Fig. 2(b) is equivalent to a non-overlapped IPI.

The collection of these equivalent non-overlapped IPIs of pinhole 1 of the entire spiral orbit scan is sufficient to reconstruct a portion of the object inside the blue region, making the reconstructed image similar to that of the single pinhole baffle system. In other words, within that portion of the blue region, $V=V_{0}$, no artifact is expected. Thus, in principle, such a baffle-window design makes it possible for us to image any axial portion of interest of a very long object with projection overlaps 
to increase sensitivity.

2 It is not difficult to see that the above baffle-window design can also apply to a multiple-circle orbit - the orbit that comprises multiple equally spaced circular sub-orbits of the same size and on the same rotation axis, to produce the extended type-II artifact-free projection overlaps. All we need is that "the pitch" of the multiple-circle orbit (by "the pitch", we mean the distance between the planes of the adjacent circular sub-orbits) be equal to the distance of the adjacent pinholes.

In the following sections, simulations are performed to numerically validate the extended type-II artifact-free projection overlaps based upon the axially aligned multi-pinhole baffle-window SPECT systems illustrated in Fig. 2.

A digital modified Defrise phantom was used for all the simulations. It was a cylindrical phantom $156 \mathrm{~mm}$ in length with a diameter of $30 \mathrm{~mm}$ - an axially very long phantom. The phantom was

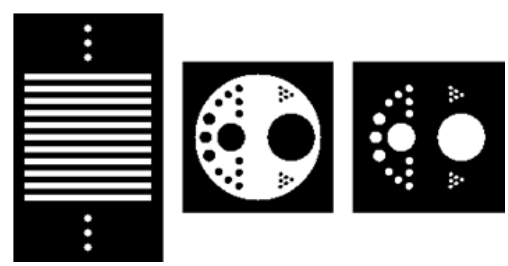

Fig. 3. The digital modified Defrise phantom used in the simulations. The sagittal plane of the modified Defrise phantom (left) is identical to that of the Defrise phantom, but the active disc (middle) and the inactive disc (right) of the modified Defrise phantom contain the same but inverted pattern.

We simulated a pixelated crystal array detector of size $102.5 \mathrm{~mm}$ (transaxial direction) by 102.5 $\mathrm{mm}$ (axial direction). The pixel size was $1.46 \mathrm{~mm}$, which lead to a pixelated crystal array of $70 \times 70$. The pinhole size was $0.7 \mathrm{~mm}$ and the acceptance angle was $60^{\circ}$. The focal distance (the distance from the pinhole to the detector) was $90 \mathrm{~mm}$, and the RoR (radius of rotation, defined as the distance from the pinhole to the axis of rotation) was $36.18 \mathrm{~mm}$.

Reconstructions were performed using the ordered subset expectation maximization (OSEM) algorithm. $^{7}$ A voxel-driven projector that models finite pinhole size as well as the detector response was used. ${ }^{5}$ Photon attenuation and scattering were not modelled. 
When the baffle window was applied as shown in Fig. 2, plane baffles were placed in between the object and the collimator. The plane baffles were parallel to the plane sensor, at a distance $20 \mathrm{~mm}$ from the axis of rotation. The axial window size produced by the baffles (the axial size of the blue region in Fig. 2) was $36 \mathrm{~mm}$, which spanned from $y=-18 \mathrm{~mm}$ to $y=18 \mathrm{~mm}$. Note that the window was in a fixed axial position relative to the phantom during the entire scan.

The corresponding 1-pinhole system, used as the benchmark of artifact-free reconstruction, was simulated for each of axially-aligned multi-pinhole systems tested.

Noiseless reconstructions were performed to investigate the existence of artifacts in the reconstructed images. Mean square error was used as the metric to compare the multiplexing systems with the non-multiplexing 1-pinhole system.

The corresponding noisy reconstructions were also performed as artifact-free reconstruction validations. Poisson noise was added to the projection data a count level of 70,000 counts per view for the 1-pinhole situation. The count level was selected based on realistic noisy phantom studies. ${ }^{4}$

To eliminate the effects of converging speed for noisy reconstructions, a post-smoothed maximum likelihood expectation maximization approach ${ }^{3,6}$ was employed. That is, a large number (300) of iterations were performed to obtain a converged image first. Then, a post smoothing filter was applied to compromise the noise and resolution. The FWHM of the applied Gaussian smoothing filter was $0.75 \mathrm{~mm}$. The noise amplitude (standard deviation) vs. the edge contrast as a function of the iteration number is also provided.

\section{A. Simulations with Spiral Orbit}

The pitch of the spiral orbit was chosen to be $12 \mathrm{~mm}$. Five turns were used for the spiral orbit, which led to an axial range of $60 \mathrm{~mm}$. The axial span of the spiral orbit ranged from $y=-30 \mathrm{~mm}$ to $y=30 \mathrm{~mm}$. There were altogether 300 projections along the spiral orbit, i.e. 60 projections each turn. Seventy-five subsets were used for the OSEM algorithm for fast convergence.

First, an axially aligned 3-pinhole system was tested. The three pinholes were uniformly distributed with the middle pinhole on the scan orbit. The adjacent pinhole distance was $12 \mathrm{~mm}$, i.e. equal to the pitch of the spiral orbit, to produce the extended type-II artifact-free overlaps when the baffle window was applied.

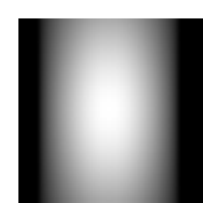

(a)

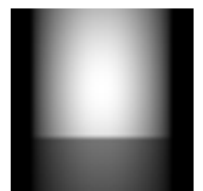

(b)

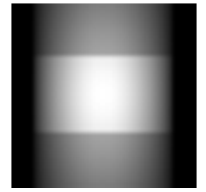

(c)

Fig 4. Illustration of the amount of projection overlap of the 3-pinhole system with baffles applied. The detector is at $y=0 \mathrm{~mm}$ (midpoint of the spiral orbit), and the axis of rotation is in the vertical direction. A uniform phantom is used to show the overlaps. (a) The IPI from the center pinhole only. (b) Two IPIs from the center pinhole and one side 
pinhole. (c) All three IPIs from the 3 pinholes.

Simulations were performed with and without the baffle window for comparison. With the baffle window applied, the axial range of the spiral orbit $(60 \mathrm{~mm})$ symmetrically covered the $36 \mathrm{~mm}$ axial window produced by the baffles with an extra of $12 \mathrm{~mm}$ on both sides. As shown in Fig. 4, the amount of overlap was quite substantial for the baffle windowed 3-pinhole system. However, since the extended type-II artifact-free overlaps were produced from the design, a portion inside the windowed region $(-18 \mathrm{~mm}<y<18 \mathrm{~mm})$ was expected to be reconstructed with no artifact.

Without the baffle window, no non-overlapped region existed since the phantom was too long (156 $\mathrm{mm})$ and the axial range of the spiral orbit was not long enough $(60 \mathrm{~mm})$. The overlaps were not artifact-free projection overlaps. Empirically, artifacts were expected.

In the above design, we chose the pitch of the orbit to be equal to the adjacent pinhole distance (12 $\mathrm{mm})$. This is critical in producing the extended type-II artifact-free overlaps as shown in the Theory section, because it guarantees that in Fig. 2(b) for overlap $\mathrm{C}^{\prime} \mathrm{B}^{\prime}$ the projection of $V$ through pinhole 2 is equal to the projection of $V_{0}$ through pinhole 2 . In a real implementation, no matter how precise the built system is, there is an error. Thus, we need to test whether the system tolerates small differences between the pitch and the adjacent pinhole distance.

Intuitively, small errors would not lead to a problem. Imagine the case where two objects $V_{0}$ and $V$ $\left(V \neq V_{0}\right)$ have an identical projection in a particular direction. When the direction deviates from that particular direction, the two projections of the two objects also change. However, we observe the following fact (though not proven): when the deviation from the particular direction approaches 0 , the difference between the two projections approaches 0 .

Our situation here is much better. The two objects $V_{0}$ and $V$ not only have the same projection in just one direction; they have the same projections along the pinhole trajectory - the entire spiral orbit! Compared with the one particular projection direction case discussed above, this has a much greater restriction on the difference between $V$ and $V_{0}$ even if they are not identical. This indicates that in many situations, the projection difference from $V$ and $V_{0}$ could be small enough to produce accurate enough approximated extended type-II artifact-free overlaps when the pitch and the adjacent pinhole distance differ. Therefore, to test this, we have purposely designed the simulation below.

An axially-aligned 4-pinhole system was tested on the spiral orbit with the baffle window applied. The four pinholes were uniformly distributed - symmetrically two above and two below the scan orbit. The adjacent pinhole distance was $8 \mathrm{~mm}$, which was purposely chosen to differ from the pitch of the spiral orbit. Apparently, the amount of overlap in the 4-pinhole system is even larger than the 3-pinhole system.

\section{B. Simulations with Multiple-circle Orbit}

A multiple-circle orbit is commonly seen in pinhole SPECT. ${ }^{8,9}$ In practice, when the pitch of the multiple-circle orbit (the distance between the planes containing two adjacent circular sub-orbits) is 
reasonably small, the 3-D volume of the imaged object can be reconstructed quite well. Therefore, multiple-circle orbits were also tested.

The same 3-pinhole collimator used earlier, i.e. the pinholes axially uniformly aligned with the middle pinhole on the scan orbit and adjacent pinhole distance being $12 \mathrm{~mm}$, was employed, and the baffle window was applied to produce the extended type-II artifact-free projection overlaps.

First, orbit MC5, i.e. the multiple-circle orbit comprising five circles, was tested. The pitch of orbit MC5 was $12 \mathrm{~mm}$, which was equal to the distance of the adjacent pinholes. Thus, the orbit MC5 situation precisely produced the extended type-II artifact-free overlaps. The five planes containing the five circular sub-orbits were: $y=-24 ; y=-12 ; y=0 ; y=12$; and $y=24$. There were altogether 300 projections for the MC5 orbit, i.e. 60 projections for each of the five circular sub-orbits. Sevetny-five subsets were used for the OSEM algorithm for fast convergence.

Next, orbit MC7, i.e. the multiple-circle orbit comprising seven circles, was designed to test the tolerance on the difference between the orbit pitch and the adjacent pinhole distance. The pitch of orbit MC7 was $8 \mathrm{~mm}$, which differed from the $12 \mathrm{~mm}$ adjacent pinhole distance. The seven planes containing the seven circular sub-orbits were: $y=-24 ; y=-16 ; y=-8 ; y=0 ; y=8 ; y=16$; and $y=$ 24. There were altogether 294 projections for the MC7 orbit, i.e. 42 projections for each of the seven circular sub-orbits. Forty-nine subsets were used for the OSEM algorithm for fast convergence.

\section{RESULTS}

\section{A. Simulations with Spiral Orbit}

In Fig. 5(a), the mean square errors (MSE) of the baffled-windowed region as a function of the iteration number for the non-multiplexing 1-pinhole system, the multiplexing 3-pinhole system with and without baffles, and the multiplexing 4-pinhole system with baffles, are plotted. Results show that when the reconstructed images converge, the MSEs of the two baffle-windowed multiplexing systems are of magnitudes very similar to that of the non-multiplexing 1-pinhole system, quantitatively validating that these baffle-windowed systems are artifact-free. On the other hand, the MSE of the 3-pinhole system with no baffle is considerably larger, suggesting that artifacts exist in the reconstructed image. The MSE test results confirm the theoretical predictions of the designs.

In Fig. 6 and Fig. 7, noiseless reconstructed images on the 1-pinhole system with and without the baffle window applied are shown as references for comparisons. The profiles of the vertical central line of the left image in Figs 6(b) and 7(b) (the Defrise phantom pattern) are shown in Fig. 8(a) and 8 (b). When there is no baffle window (Fig. 6), the axial range of the reconstructable portion should be at least $4 \times 12=48 \mathrm{~mm}$, given the spiral orbit has 5 turns and the pitch of the spiral orbit is 12 $\mathrm{mm}$. As expected, the results show a large axial portion reconstructed with no artifact. With the baffle window applied, one expects a well-reconstructed fraction inside the windowed portion $(-18 \mathrm{~mm}<y<18 \mathrm{~mm}$, indicated by the red dashed lines). In fact, the entire windowed portion $(-18 \mathrm{~mm}<y<18 \mathrm{~mm})$ was reconstructed reasonably well, due to the small pitch of the spiral orbit.

In Fig. 9, noiseless reconstructed images of the axially aligned 3-pinhole system with no baffle are 
shown. The profile is shown in Fig. 8(c). Consistent with the MSE result shown earlier, severe

2 artifacts associated with the overlaps were observed in the reconstructed image.

3 Fig. 10 shows the noiseless reconstructed images of the 3-pinhole system with the baffle window 4 applied. The profile is shown in Fig. 8(d). The existence of the extended type-II artifact-free 5 overlaps leads to a fraction inside the windowed portion, $-18 \mathrm{~mm}<y<18 \mathrm{~mm}$ indicated by the red 6 dashed lines, to be reconstructed without artifacts. Again, the entire windowed portion, $-18 \mathrm{~mm}<y$ $7<18 \mathrm{~mm}$, was reconstructed reasonably well, due to the small pitch of the spiral orbit.

8 Fig. 11 depicts the noiseless reconstructed images of the 4-pinhole system with the baffle window 9 applied. The profile is shown in Fig. 8(e). This system was designed to test the tolerance on the difference between the orbit pitch and adjacent pinhole distance. Consistent with MSE test results, reconstructed images of the 4-pinhole system here are very similar to that of the 3-pinhole system in Fig. 10 where the extended type-II artifact-free overlaps were generated precisely.
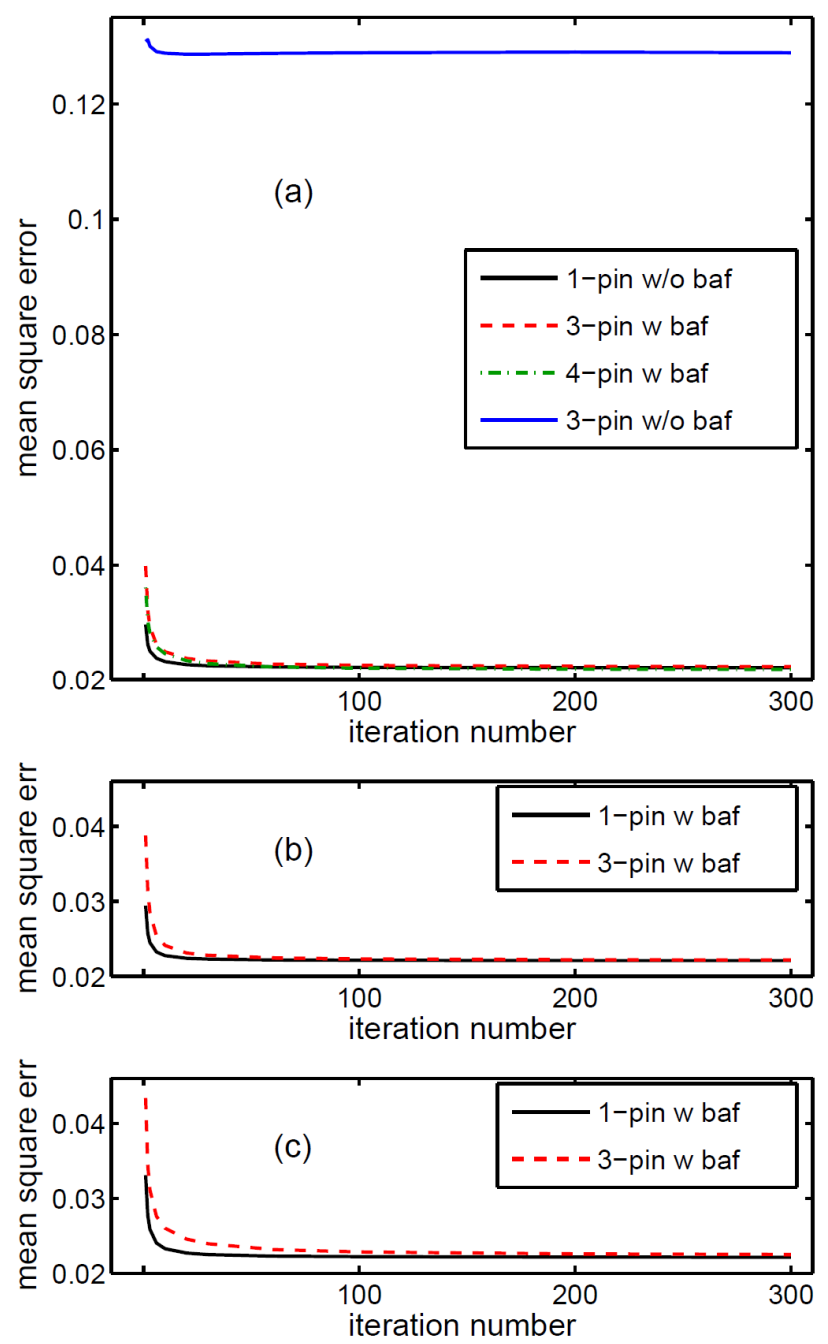

Fig. 5. (a) Spiral orbit MSE test results: MSE of the baffled-windowed region as a function of the iteration number for the non-multiplexing 1-pinhole system, the multiplexing 3-pinhole system with and without baffles, and the multiplexing 4-pinhole system with baffles; (b) MC5 orbit MSE test results: MSE for the non-multiplexing 1-pinhole system and the multiplexing 3-pinhole system with baffles; (c) MC7 orbit MSE test results: MSE for the nonmultiplexing 1-pinhole system and the multiplexing 3-pinhole system with baffles. 
(a)

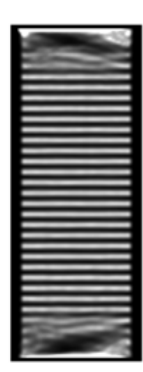

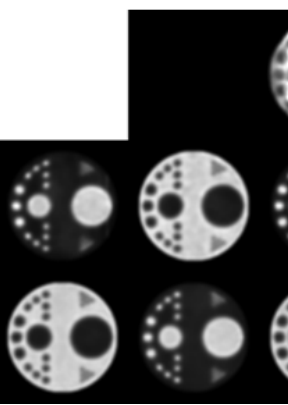

(19) से $60 \div 96$ 6909

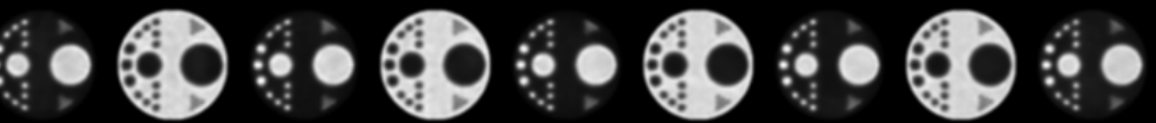
$9 \% 90 \% 0 \%$

(b)

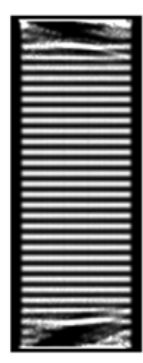

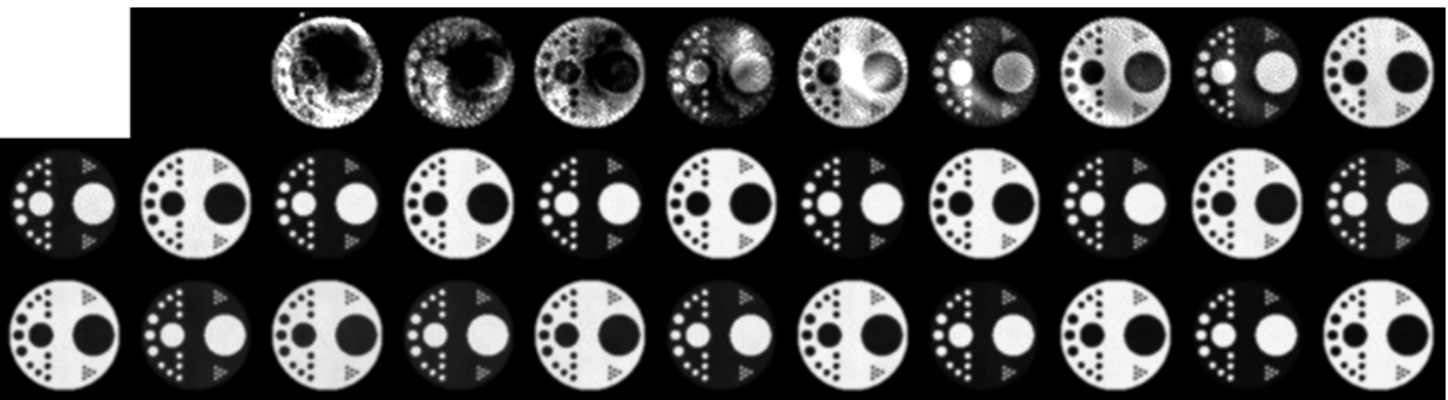

Fig. 6. Simulation results for the 1-pinhole system with no baffle window applied: (a) The reconstructed image at iteration 1; and (b) The reconstructed image at iteration 300. On the left of each sub-figure, the sagittal plane (same pattern as the Defrise phantom) of the reconstructed image is shown. On the right, cross section slices for each active and inactive disc, starting from the edge of the reconstructed volume to the central disc, are shown. The order of the shown discs is "left to right and top to bottom". (Symmetrically, the other half of the discs of the reconstructed volume is not shown to save space.)

(a)

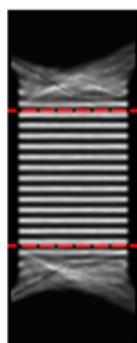

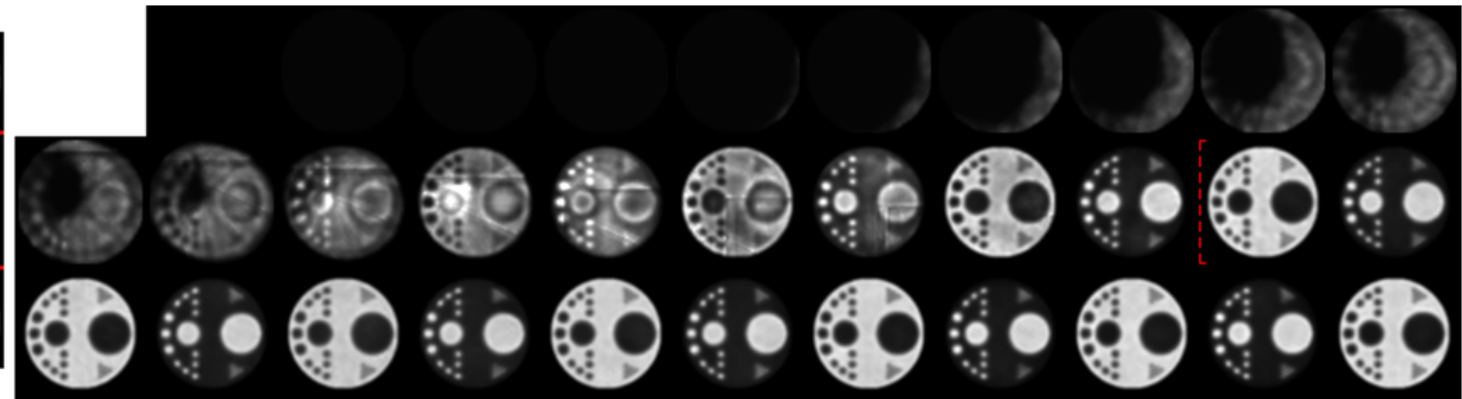

(b)

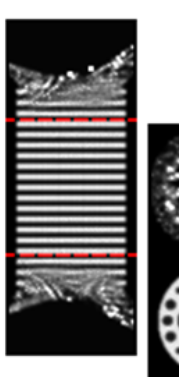

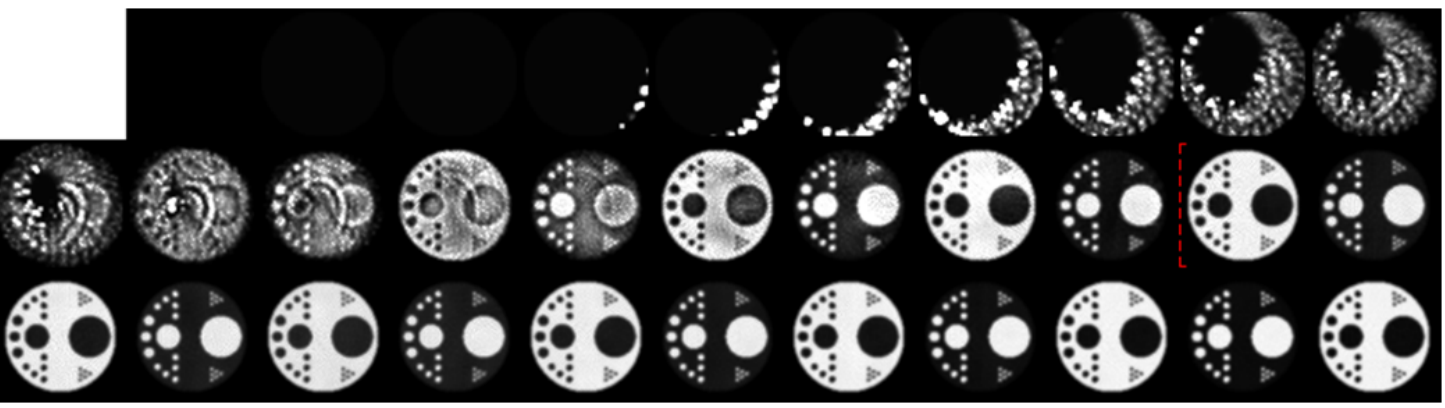

Fig. 7. Simulation results for the 1-pinhole system with baffle window applied: (a) The reconstructed image at iteration 1; and (b) The reconstructed image at iteration 300 . The region enclosed by the red dashed lines is the baffle windowed region $(-18 \mathrm{~mm}<y<18 \mathrm{~mm})$; i.e. corresponding to the blue region in Fig. 2. 

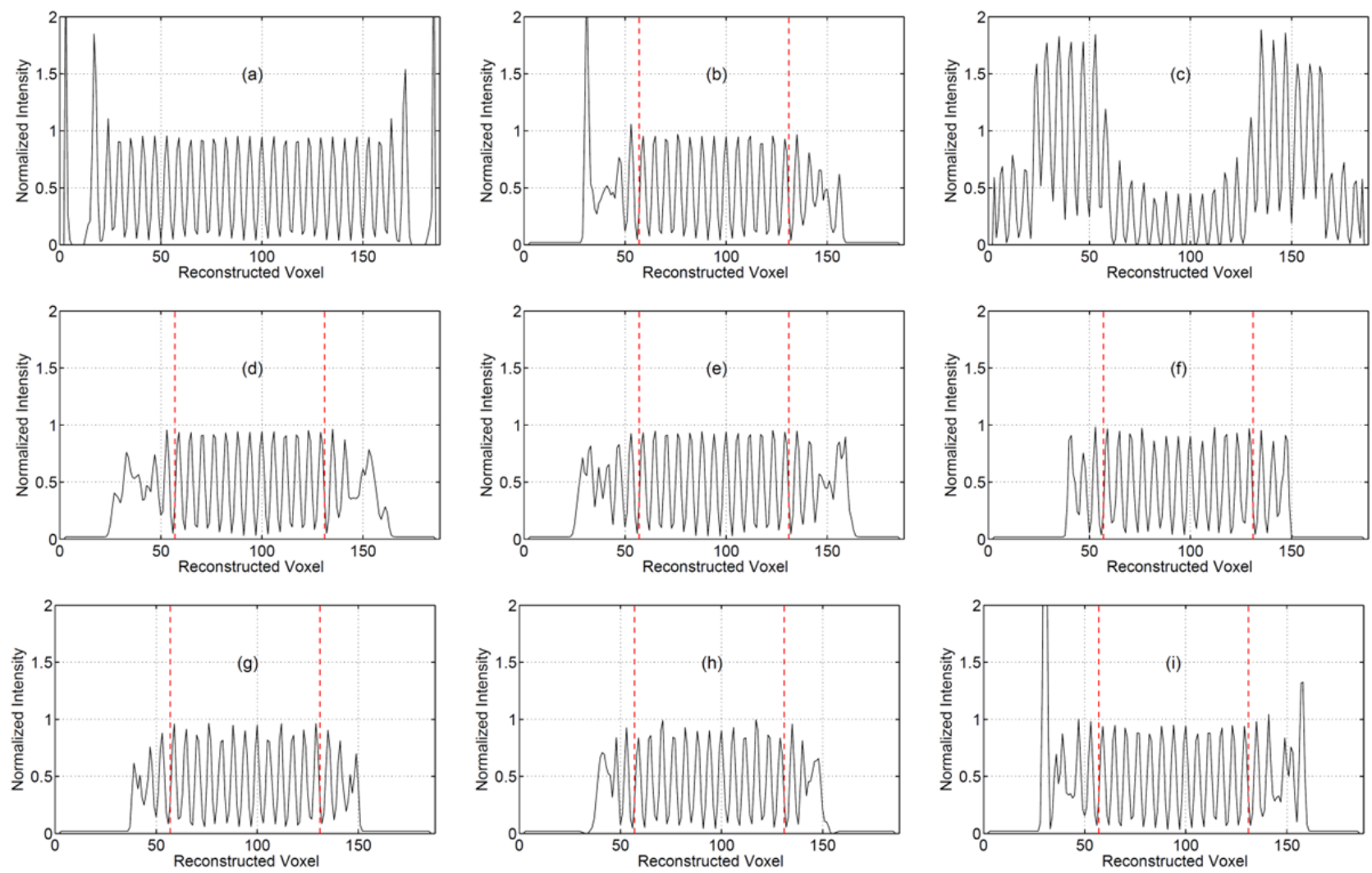

Fig. 8. Profiles along the vertical central line of the left images (the Defrise phantom pattern) in Figs 6, 7, 9, 10, 11, and 14: (a) for Fig. 6(b); (b) for Fig. 7(b); (c) for Fig. 9(b); (d) for Fig. 10(b); (e) for Fig. 11(b); (f) for Fig. 14(a); (g) for Fig. 14(b); (h) for Fig. 14(c); and (i) for Fig. 14(d).

5

(a)
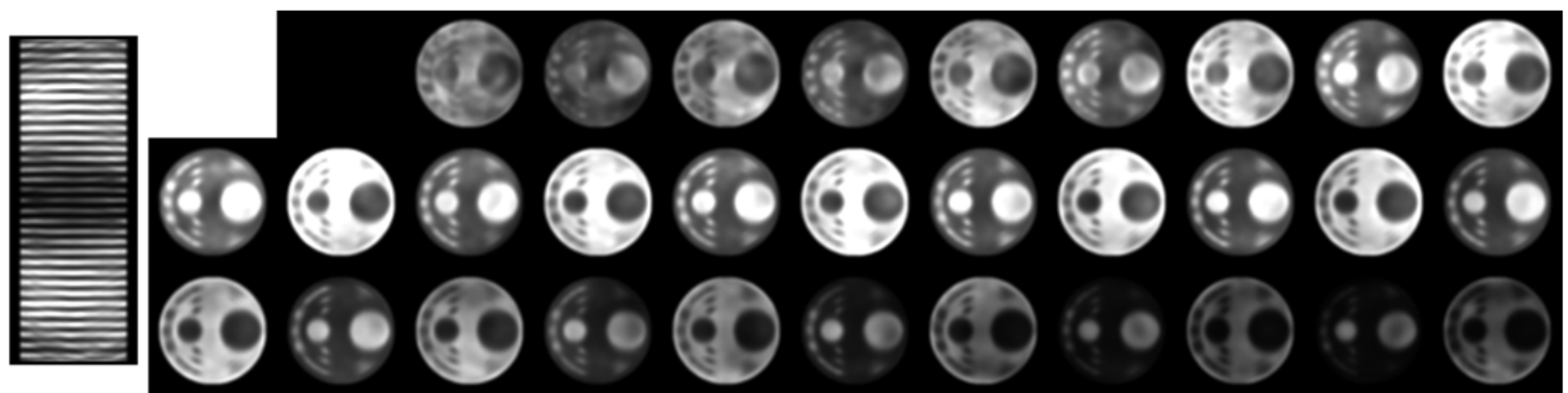

(b)
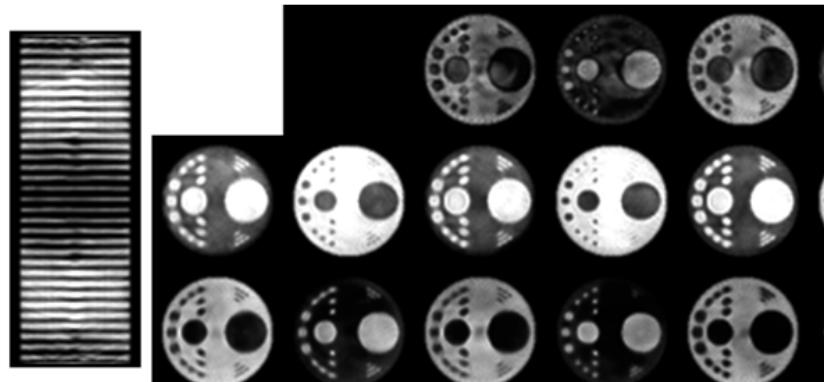

6069 606960 웅 $\circ 9$ 웅

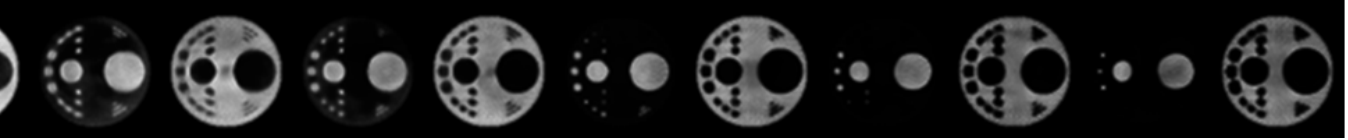

Fig. 9. Simulation results for the 3-pinhole system with no baffle window applied: (a) The reconstructed image at iteration 1; and (b) The reconstructed image at iteration 300. 


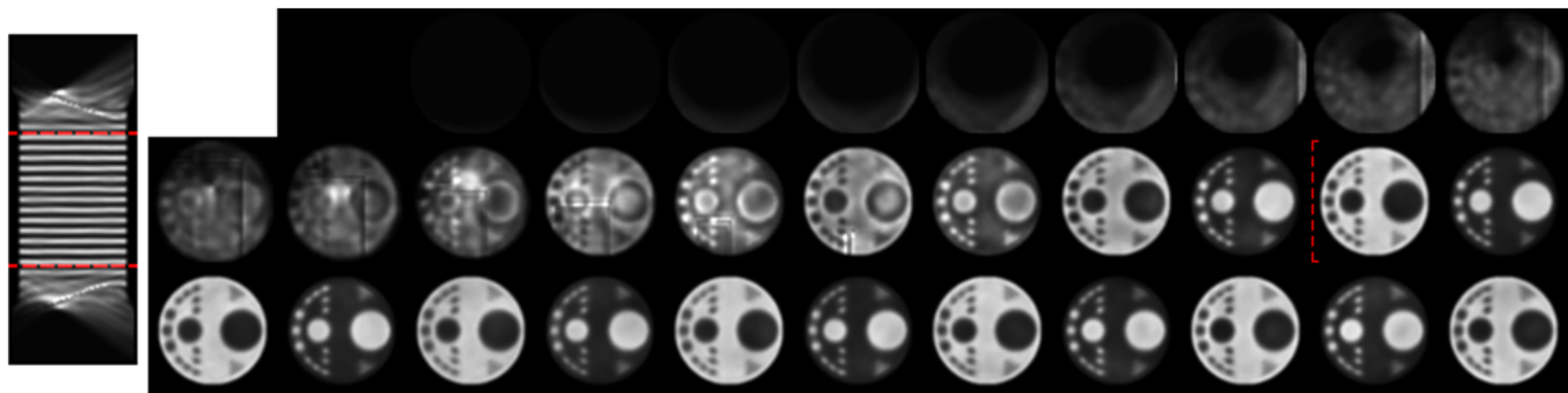

(b)
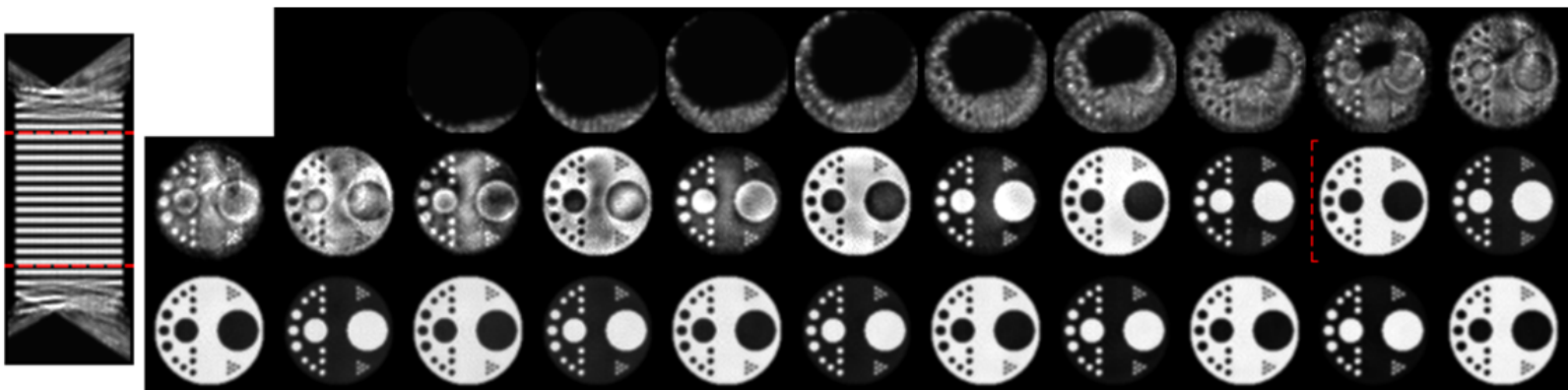

Fig. 10. Simulation results for the 3-pinhole system with baffle window applied: (a) The reconstructed image at iteration 1; and (b) The reconstructed image at iteration 300.

(a)

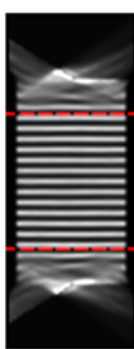

6 (b)

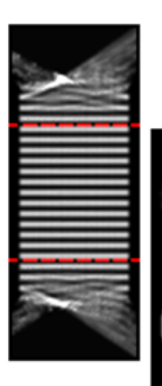

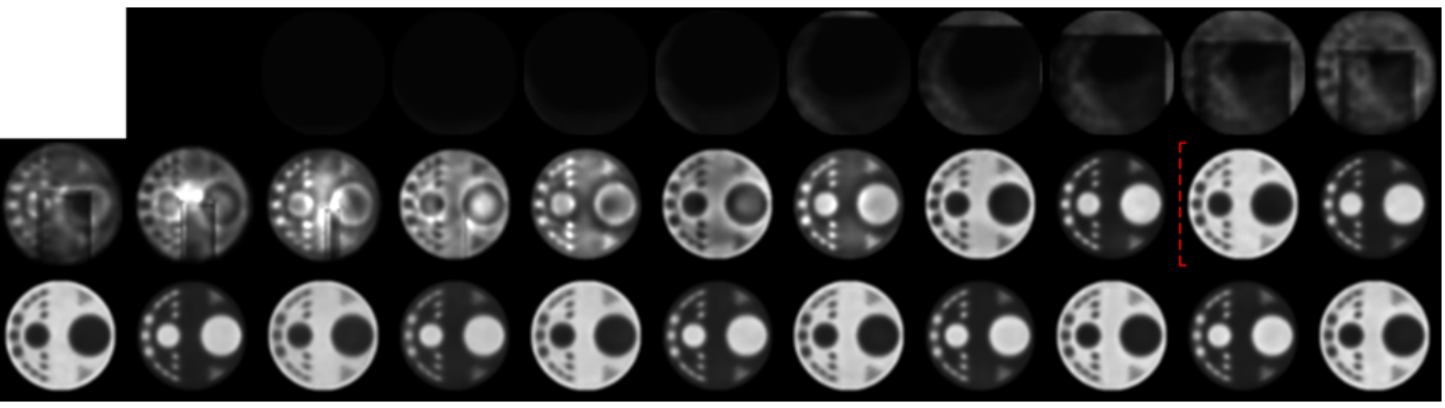
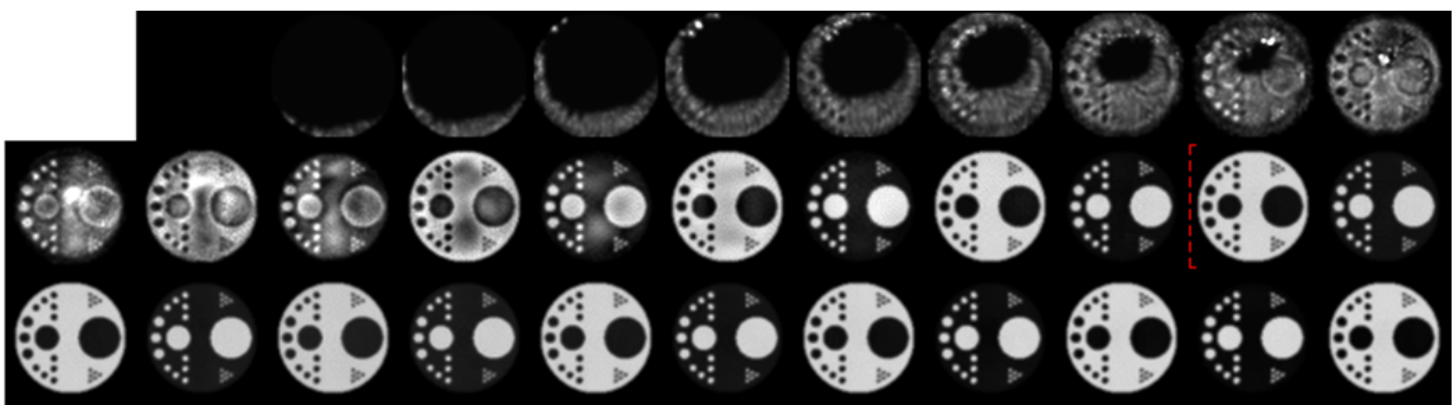

Fig. 11. Simulation results for the 4-pinhole system with baffle window applied: (a) The reconstructed image at iteration 1; and (b) The reconstructed image at iteration 300.

Fig. 12 shows the reconstructed images for the spiral orbit systems using noisy projections. As expected, the results are consistent with their corresponding noise-free simulations; i.e., no artifact is observed within the windowed portion $-18 \mathrm{~mm}<y<18 \mathrm{~mm}$ for the 3-pinhole and the 4-pinhole multiplexing systems with baffles. Besides, visually one can observe that the 3-pinhole and the 4pinhole multiplexing systems lead to lower noise and thus better quality for their reconstructed images compared with the 1-pinhole systems. To quantitatively show the noise property during 
1 reconstruction, the noise amplitude vs. the contrast at edges at iteration 1,2,3,6,10,20,30,60, and 2 100, is plotted in Fig. 13(a).

3

(a)

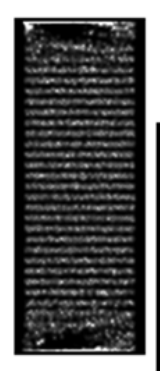

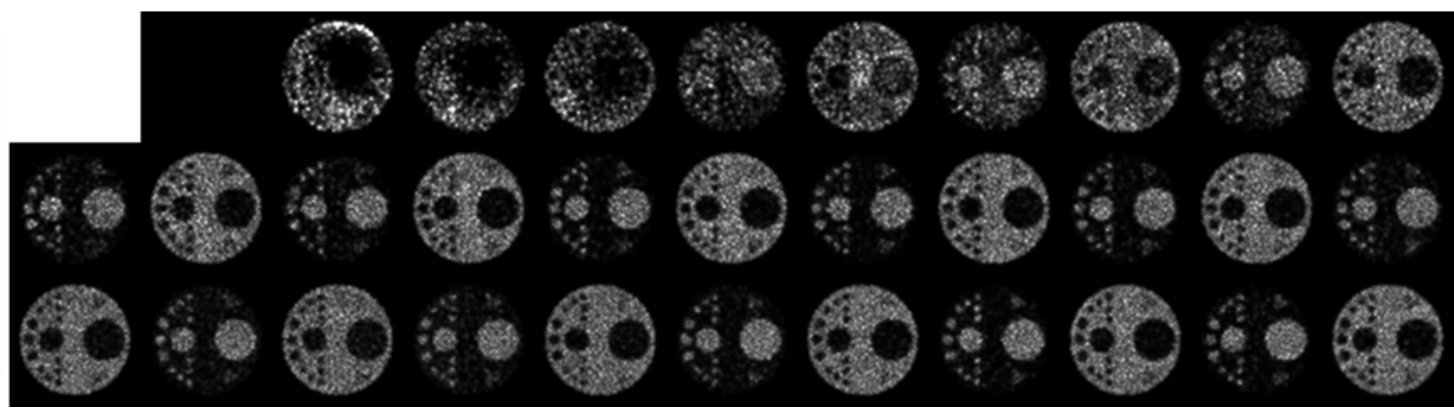

4

(b)

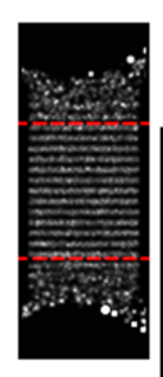

5

(c)
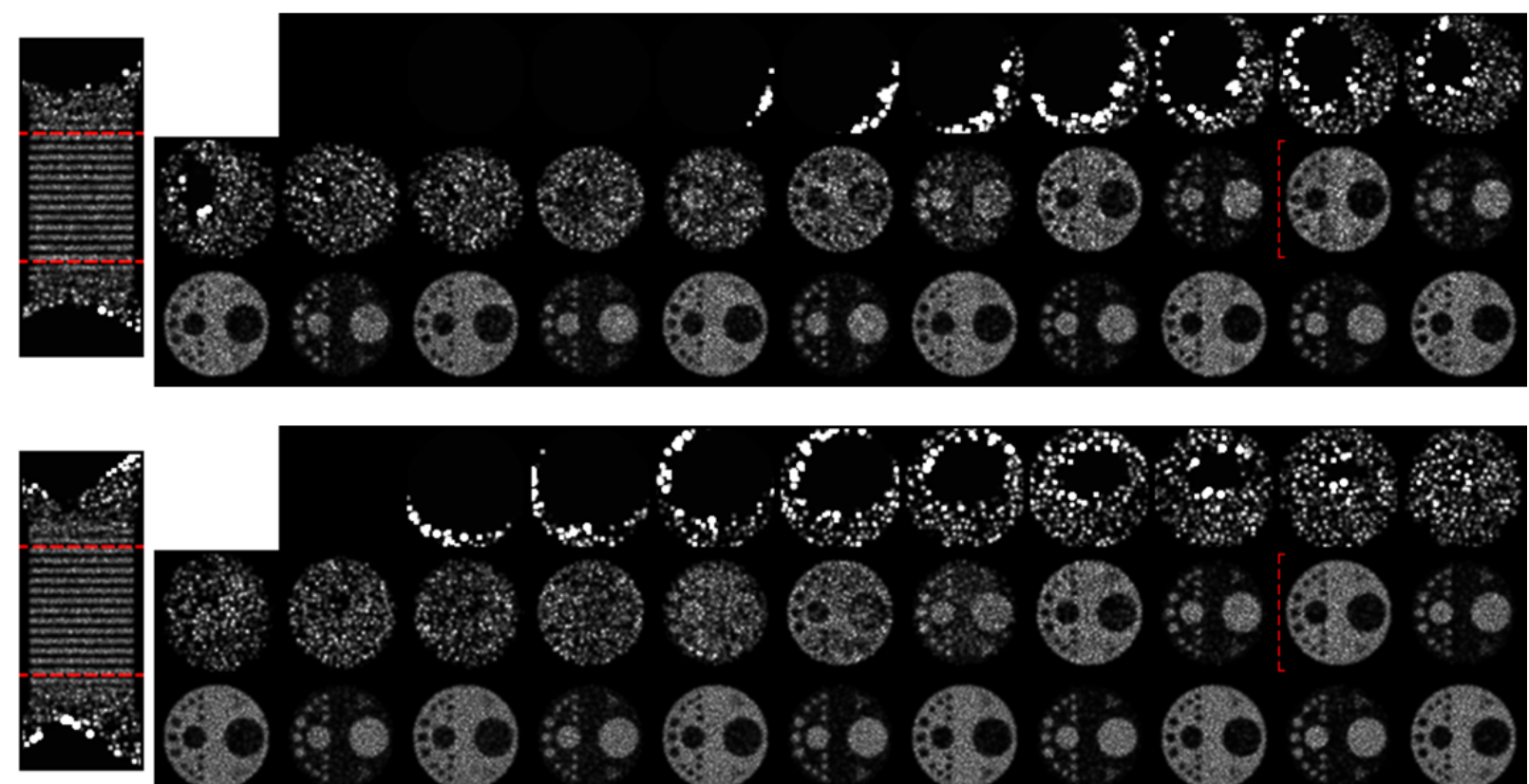

6

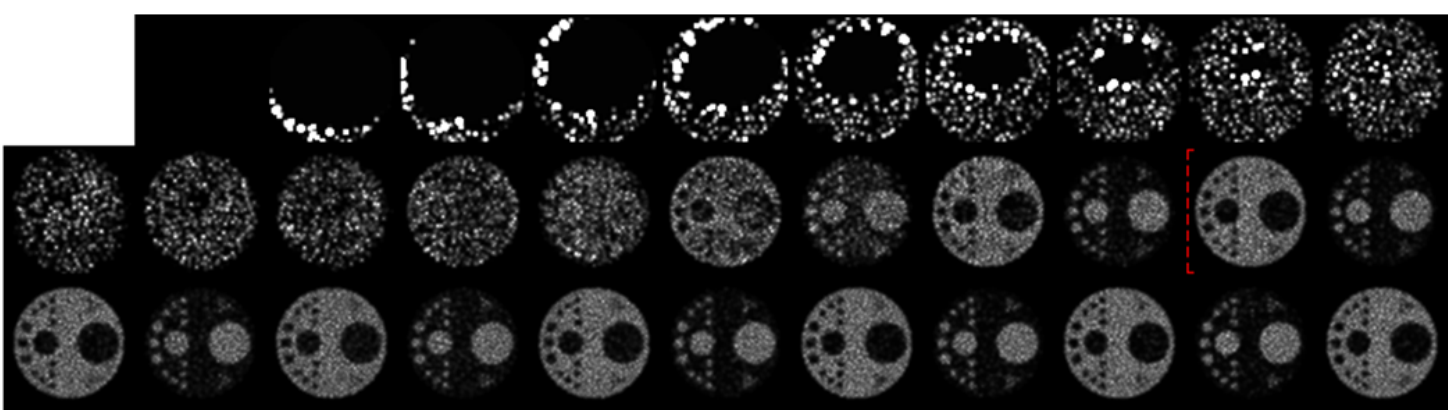

(d)
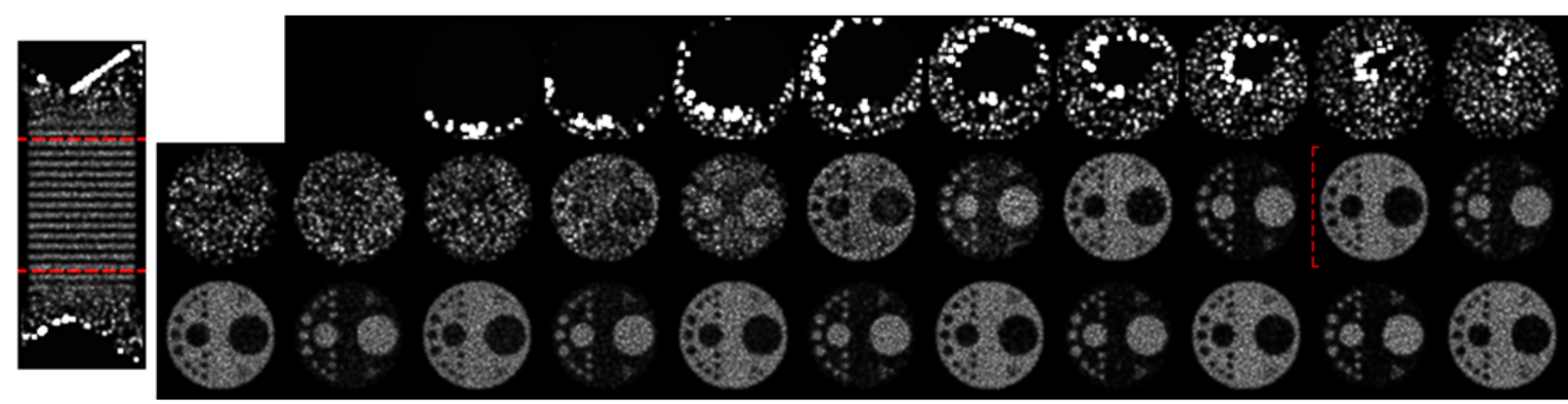

Fig. 12. Simulation results for the spiral orbit systems using noisy projections. All the reconstructed images shown were window applied; (c) The 3-pinhole system with the baffle window applied; and (c) The 4-pinhole system with the baffle window applied. 

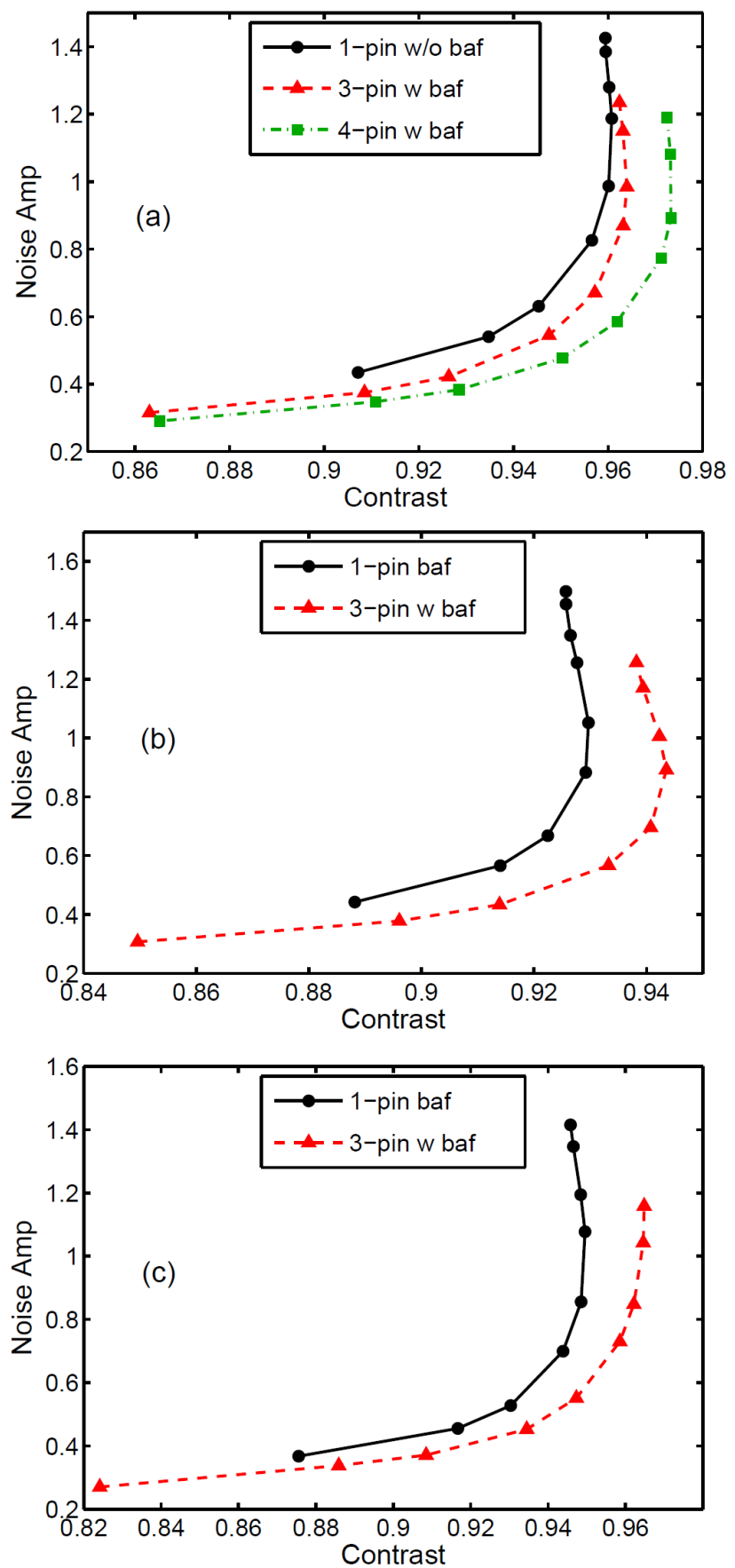

Fig. 13. Noise amplitude vs. the edge contrast at iterations 1, 2, 3, 6, 10, 20, 30, 60 and 100 in noisy reconstructions: (a) The spiral orbit test; (b) The MC5 orbit test; and (c) the MC7 orbit test.

\section{B. Simulations with Multiple-circle Orbit}

6 In Fig. 5(b) and 5(c), mean square error (MSE) results of the multiple circle orbit tests are shown. 7 Again, when the reconstructed images converge, the magnitudes of the MSEs of the baffle8 windowed multiplexing systems are very similar to that of the non-multiplexing 1-pinhole system, 9 quantitatively confirming that these baffle-windowed systems are artifact-free.

In Fig. 14, noiseless reconstructed images for the multiple-circle orbits are shown. The corresponding profiles are shown in Fig. 8(f), 8(g), 8(h) and 8(i). For orbit MC5, when comparing 
1 Fig. 14(b), the 3-pinhole system with the extended type-II artifact-free overlaps, with Fig. 14(a), the 2 1-pinhole system, no significant difference is observed within the baffle windowed region, $-18 \mathrm{~mm}$ $3<y<18 \mathrm{~mm}$ indicated by the red dashed lines, as expected.

4 For orbit MC7, when comparing the 3-pinhole system with the 1-pinhole system, again, no 5 significant difference is observed within the baffle windowed region.

6

(a)

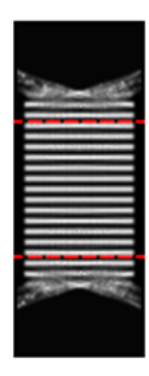

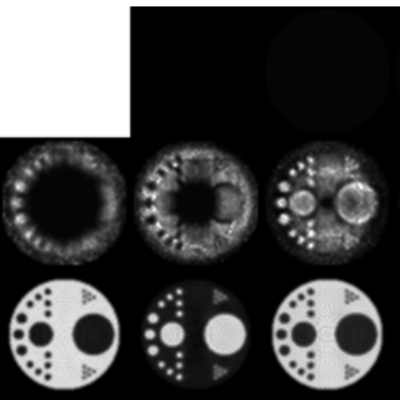

(b)

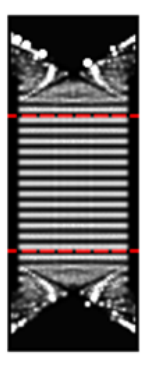

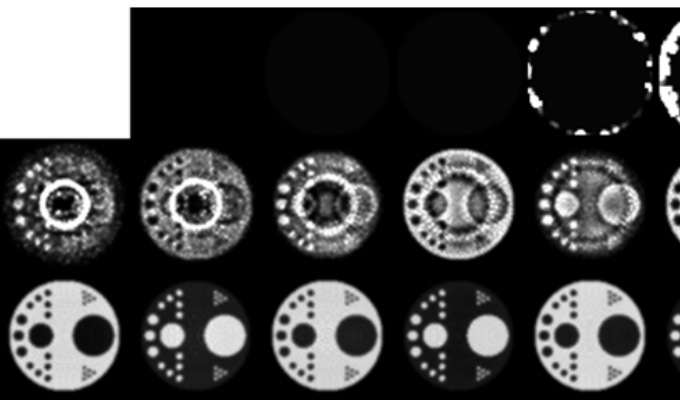
0 090

(c)

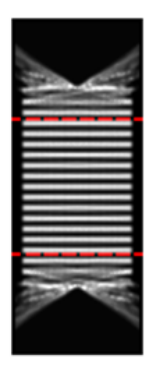

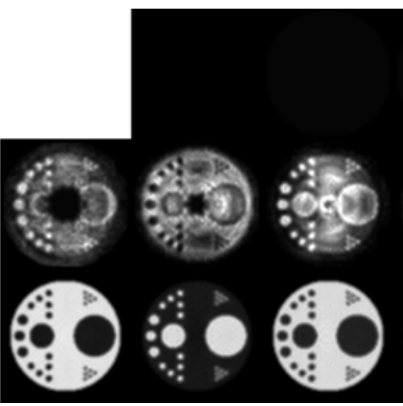
06
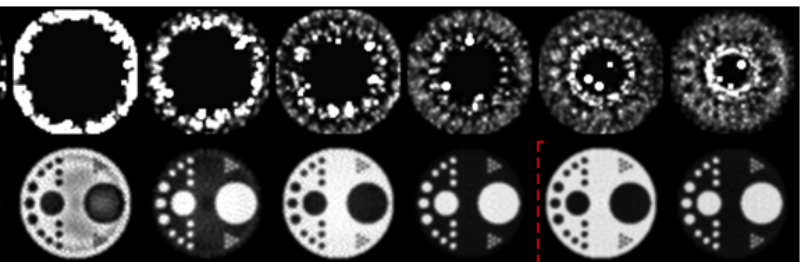

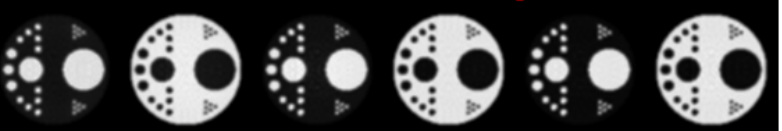

(d)

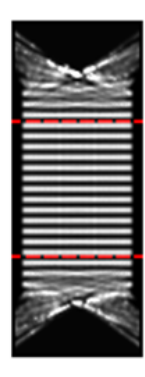

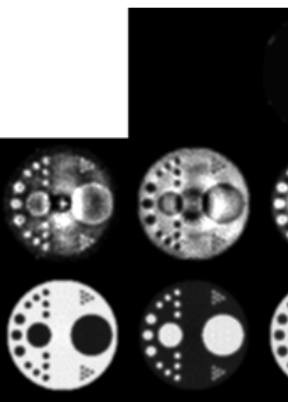
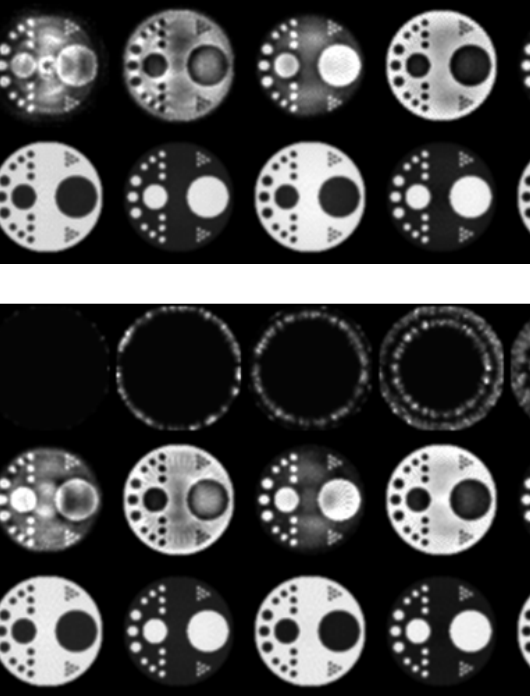

ใด

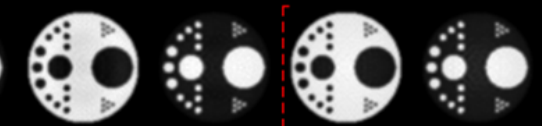

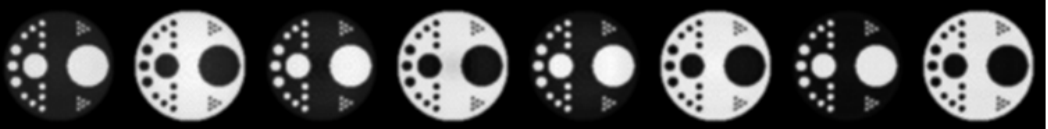

Fig. 14. Simulation results for systems tested on the multiple-circle orbits MC5 and MC7 with the baffle window applied. All the reconstructed images shown were reconstructed with 300 iterations: (a) The 1-pinhole system on the MC5 orbit; (b) The 3-pinhole system on the MC5 orbit; (c) The 1-pinhole system on the MC7 orbit; and (d) The 3pinhole system on the MC7 orbit. 
(a)

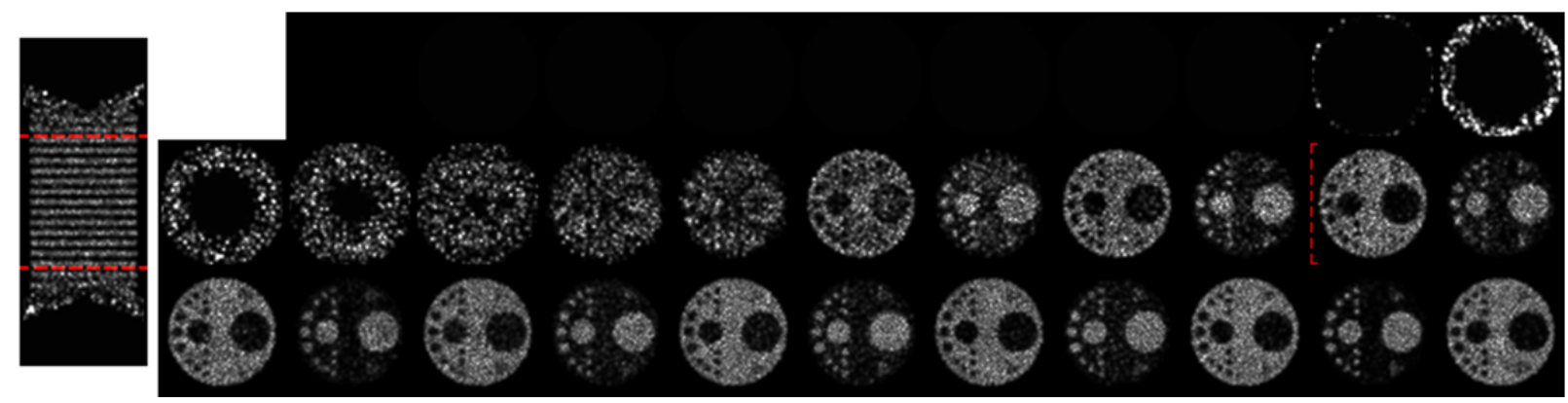

(b)

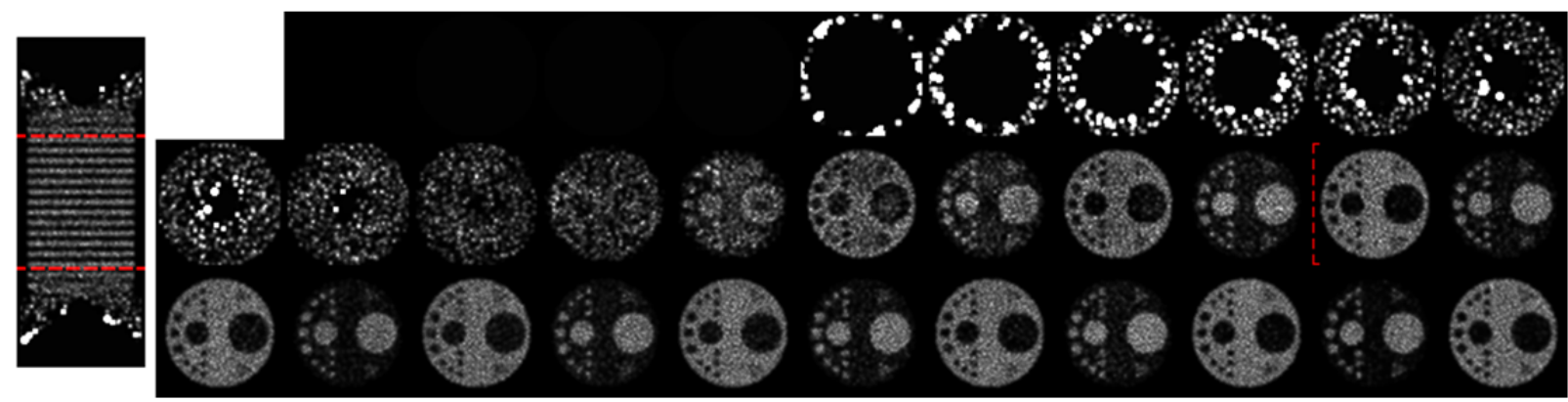

(c)
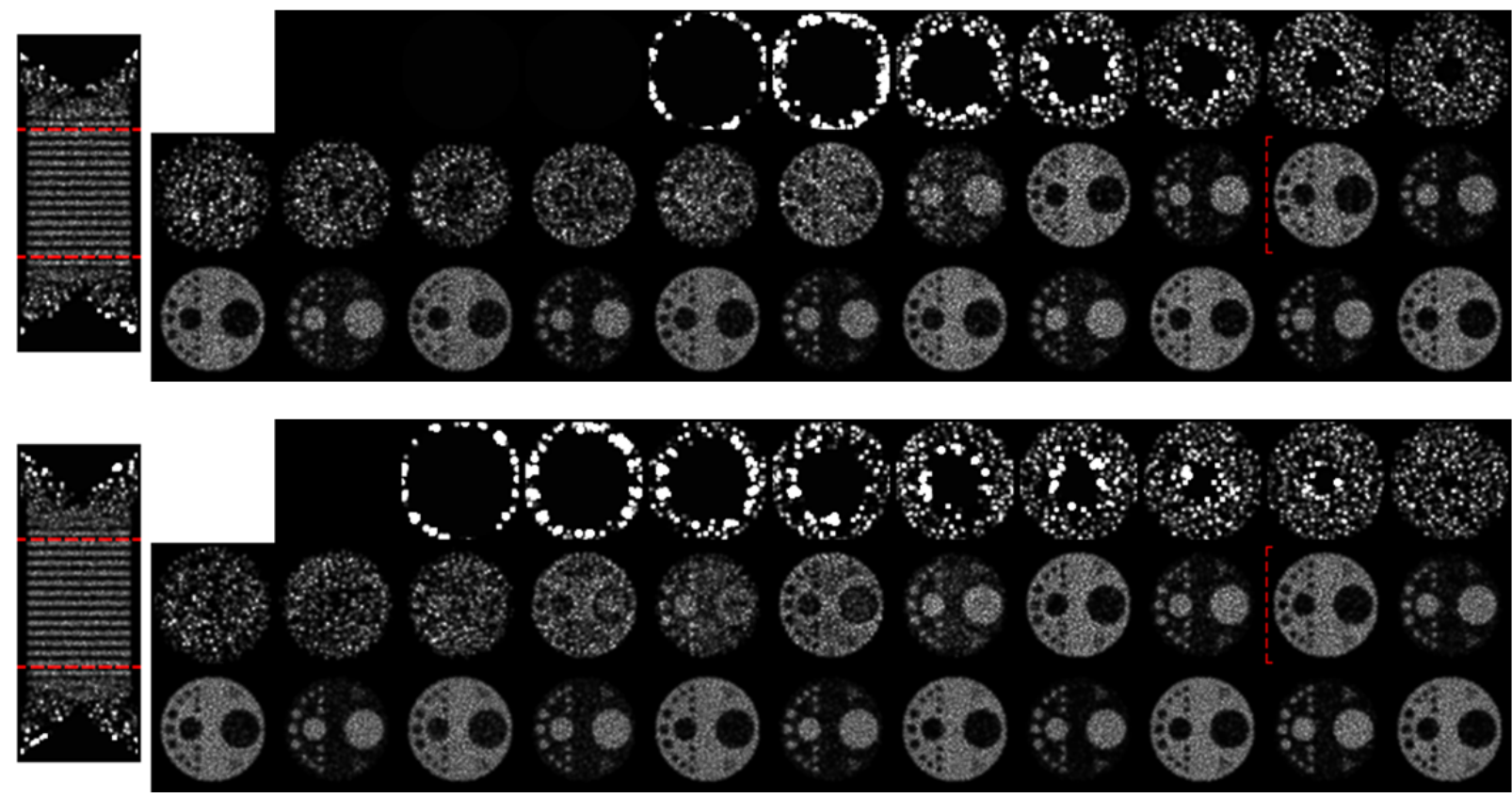

Fig. 15. Simulation results using noisy projections for multiple-circle orbits with the baffle window applied: All the reconstructed images shown were reconstructed with 300 iterations: (a) The 1-pinhole system on the MC5 orbit; (b) The 3-pinhole system on the MC5 orbit; (c) The 1-pinhole system on the MC7 orbit; and (d) The 3-pinhole system on the MC7 orbit.

In Fig. 15, reconstructed images using noisy projections for the multiple-circle orbits with the baffle window applied are shown. Again, the results are consistent with the results from noise free simulations shown in Fig. 14 as expected, i.e., no artifact is observed within the windowed portion $-18 \mathrm{~mm}<y<18 \mathrm{~mm}$. Visually, one could observe that for both the MC5 orbit and the MC7 orbit, the 3-pinhole multiplexing system leads to lower noise and thus better quality for the reconstructed images compared with the 1-pinhole system. The noise amplitude vs. the contrast at edges at iteration 1, 2, 3, 6, 10, 20, 30, 60, and 100, is plotted in Fig. 13(b) for the MC5 orbit test, and Fig. 13(c) for the MC7 orbit test. 
Firstly, we would like to distinguish between artifacts and degradations. An artifact is defined here as the ambiguity associated with the non-uniqueness of the solution of Equation (1). In other words, given the projections, there are many $V$ 's that produce the same given projections, and thus, in theory it is impossible to recover $V_{0}$ mathematically.

Degradation is associated with the imperfections in real imaging and reconstruction. In reality, it is almost impossible (at least impractical) to perform a precise reconstruction even to computer precision and even for the single pinhole situation. First, the pinhole size is not infinitely small and the resolution of the sensor is not infinitely high. Second, the sampled pinhole trajectory is not continuous. Third, all the numerical computations (analytical or iterative) unavoidably use various kinds of approximations. Moreover, there are other imperfections.

This study focuses on artifacts, not on degradations associated with imperfections. However, IPI regions within artifact-free projection overlaps are, in principle, deciphered with the help from other reconstructed image portions where degradations exist. Therefore, we need to keep in mind that degradations can accumulate even if artifacts are avoided. Nevertheless, artifact-free overlaps do contribute to the final quality (noise reduction) of the reconstructed image when they are well designed. $^{3}$

In the example of the baffle window system designs, the difference between the orbit pitch and the adjacent pinhole distance, which leads to approximations in producing the extended type-II artifactfree overlaps, is considered an imperfection, because the errors approach 0 when the difference between the pitch and the adjacent pinhole distance approaches 0 . In the tolerance tests, the 4pinhole spiral orbit case and the MC7 case, the degradation associated with this imperfection is not obvious when compared with the corresponding precise implementations. This suggests that this imperfection could be less significant than other imperfections (such as discrete sampling of the pinhole orbit, finite pinhole size, finite pixel size of the projections, and approximations used in computation ... etc.). Therefore, the requirement that the pitch be equal to the adjacent pinhole distance could be relaxed in real designs.

Secondly, the converging speed of the multi-pinhole systems with overlaps is a bit slower than the 1-pinhole non-overlapping system. This can be seen by the MSE plots in Fig. 5. However, noise amplification can be avoided by using the post-smoothed MLEM (maximum likelihood expectation maximization) algorithm. ${ }^{3,6}$ One advantage of this approach is that the compromise of noise and resolution is controlled and achieved solely by the post-smoothing filter, and thus the final result with noisy data is independent of the converging speed. Also, for the artifact-free multiplexing systems tested here, their noise properties during the reconstruction process plotted in Fig. 13 show that early terminations could also be employed for these systems. Moreover, converging speed is a function of the convergence path, and there could be new algorithms achieving faster converging speeds for reconstructions with multiplexing by making use of better convergence paths. ${ }^{10}$

Finally, examining the test results in Figs. 10, 11, 14(b) and 14(d), one can easily see that regions outside the windowed portion, $y<-18 \mathrm{~mm}$ and $y>18 \mathrm{~mm}$ corresponding to the grey regions in the illustrating Fig. 2, are not correctly reconstructed, which is consistent with the theory. However, to form the extended type-II artifact-free projection overlaps, $V$ must be a solution of (1) even if 
$V \neq V_{0}$, and thus those incorrectly reconstructed portions in $V$ are critically needed in the reconstruction.

To guarantee $V$ being a solution of (1), the reconstruction volume size needs to be large enough. One may try a small reconstruction volume that is only slightly larger than the baffle windowed volume portion, i.e. the blue section in Fig. 2. Then, solution $V$ to Equation (1) does not exist, and artifacts can be observed. So, one may raise the question of how to choose a large enough size for the reconstruction volume. The simplest method is to select a volume size that includes every portion of $V_{0}$ that projects to the sensors. In this way, there is at least one solution, which is $V_{0}$ (even if your solution $V$ to Equation (1) is normally not $V_{0}$, since it is not unique).

\section{CONCLUSION}

In this paper, the previously defined type-II artifact-free projection overlap ${ }^{3}$ is extended to accommodate a wider range of conditions. The extension itself is of theoretical importance in broadening the selection range of artifact-free projection overlaps for optimizing multiplexing multi-pinhole SPECT designs. There is also an immediate application of the extension, which is the imaging of a small axial portion of a long object with the help of a baffle window applied to axiallyaligned multiplexing multi-pinhole systems. This application overcomes the limitation imposed by the original type-II artifact-free overlaps; ${ }^{3}$ that is, the entire body must be imaged in order to exploit axially-aligned multiplexing in spiral orbit imaging.

\section{References}

1. K, Vunckx, P. Suetens and J. Nuyts, "Effect of overlapping projections on reconstruction image quality in multipinhole SPECT", IEEE Trans. Med. Imag., vol. 27, pp. 972-983, July 2008.

2. S. Mahmood, K. Erlandsson, I. Cullum and B. Hutton, "Experimental results from a prototype slit-slat collimator with mixed multipexed and non-multiplexed data"," Phys. Med. Biol., vol. 56, pp. 4311-4331, June 2011.

3. J. Lin, "On Artifact-free Projection Overlaps in Multi-pinhole Tomographic Imaging", IEEE Trans. Med. Imag., vol. 32, no. 12, pp. 2215-2229, Dec. 2013.

4. J. Lin and S. R. Meikle, "SPECT Using Asymmetric Pinholes with Truncated Projections", Phys. Med. Biol., vol. 56, pp. 4103-4118, June 2011.

5. J. Lin, P. L. Kench, M.-C. Gregoire, and S. R. Meikle, "Projection Process Modelling for Iterative Reconstruction of Pinhole SPECT”, IEEE Trans. Nucl. Sci., vol. 57, no. 5, pp. 25782586, Oct. 2010.

6. K. Vunckx, D. Bequ, M. Defrise and J. Nuyts, "Single and Multipinhole Collimator Design Evaluation Method for Small Animal SPECT, IEEE Trans. Med. Imag., vol. 27, pp. 36-46, Jan. 2008.

7. H. M. Hudson and R. S. Larkin, "Accelerated image reconstruction using ordered subsets of projection data", IEEE Trans. Med. Imag., vol. 13, pp. 601-609, Dec. 1994.

8. F. J. Beekman, F. van der Have, B. Vastenhouw, A. J. van der Linden, P. P. van Rijk, J. P. Burbach and M. P. Smidt, "U-SPECT-I: a novel system for submillimeter-resolution tomography with radiolabelled molecules in mice”, J. Nucl. Med., vol. 46, pp. 1194-200, July 2005.

9. F. van der Have, B. Vastenhouw, R. M. Ramakers, W. Branderhorst, J. O. Krah, C. Ji, S. G. Staelens and F. J. Beekman, "U-SPECT-II: an ultra-high-resolution device for molecular small- 
animal imaging", J. Nucl. Med. vol. 50, pp. 599-605, Apr. 2009.

10. J. Lin and S. R. Meikle, "Truncated pinhole SPECT: Sufficient sampling criteria and applications", Nuclear Science Symposium Conference Record (NSS/MIC), 2010 IEEE, pp. 2066-2070, 2010.

11. G. S. P. Mok, B. M. W. Tsui and F. J. Beekman, "The effects of object activity distribution on multiplexing multi-pinhole SPECT", Phys. Med. Biol., vol. 56, pp. 2635-2650, Apr. 2011.

12. Z. Cao, G. Bal, R. Accorsi and P. D. Acton, "Optimal number of pinholes in multi-pinhole SPECT for mouse brain imaging - a simulation study”, Phys. Med. Biol., vol. 50, pp. 46094624, 2005.

13. G. S. P. Mok, Y. Wang and B. M. W. Tsui, "Quantification of the multiplexing effects in multipinhole small animal SPECT: a simulation study”, IEEE Trans. Nucl. Sci., vol. 56, no. 5, pp. 2636-2643, Oct. 2009.

14. H. K. Tuy, “An inversion formula for cone-beam reconstruction”, SIAM J Appl Math, vol 43, pp. 546-552, 1983.

15. B. D. Smith, "Image reconstruction from cone-beam projections: Necessary and sufficient condition and reconstruction methods", IEEE Trans Med Imaging, vol 4, pp. 14-25, 1985.

16. L. A. Shepp and Y.Vardi, "Maximum likelihood reconstruction for emission tomography", IEEE Trans Med Imaging, vol 1, pp 113-122, 1982.

17. J. Qi and R. M. Leahy, "Iterative reconstruction techniques in emission computed tomography", Phys Med Biol, vol 51, pp R541-R578, 2006.

18. S. T. Mahmood, K. Erlandsson, I. Cullum and B. F. Hutton, "The potential for mixed multiplexed and non-multiplexed data to improve the reconstruction quality of a multi-slit-slat collimator SPECT system", Phys. Med. Biol., vol. 55, pp. 2247-2268, Mar. 2010.

19. S. R. Meikle, R. R. Fulton, S. Eberl, M. Dahlbom, K.-P. Wong, and M. J. Fulham, "An investigation of coded aperture imaging for small animal SPECT”, IEEE Trans. Nucl. Sci., vol. 48, no. 3, pp. 816-821, Jun. 2001.

20. F. J. Beekman and B. Vastenhouw, "Design and simulation of a high-resolution stationary SPECT system for small animals", Phys. Med. Biol., vol. 49, no. 19, pp. 4579-4592, 2004. 